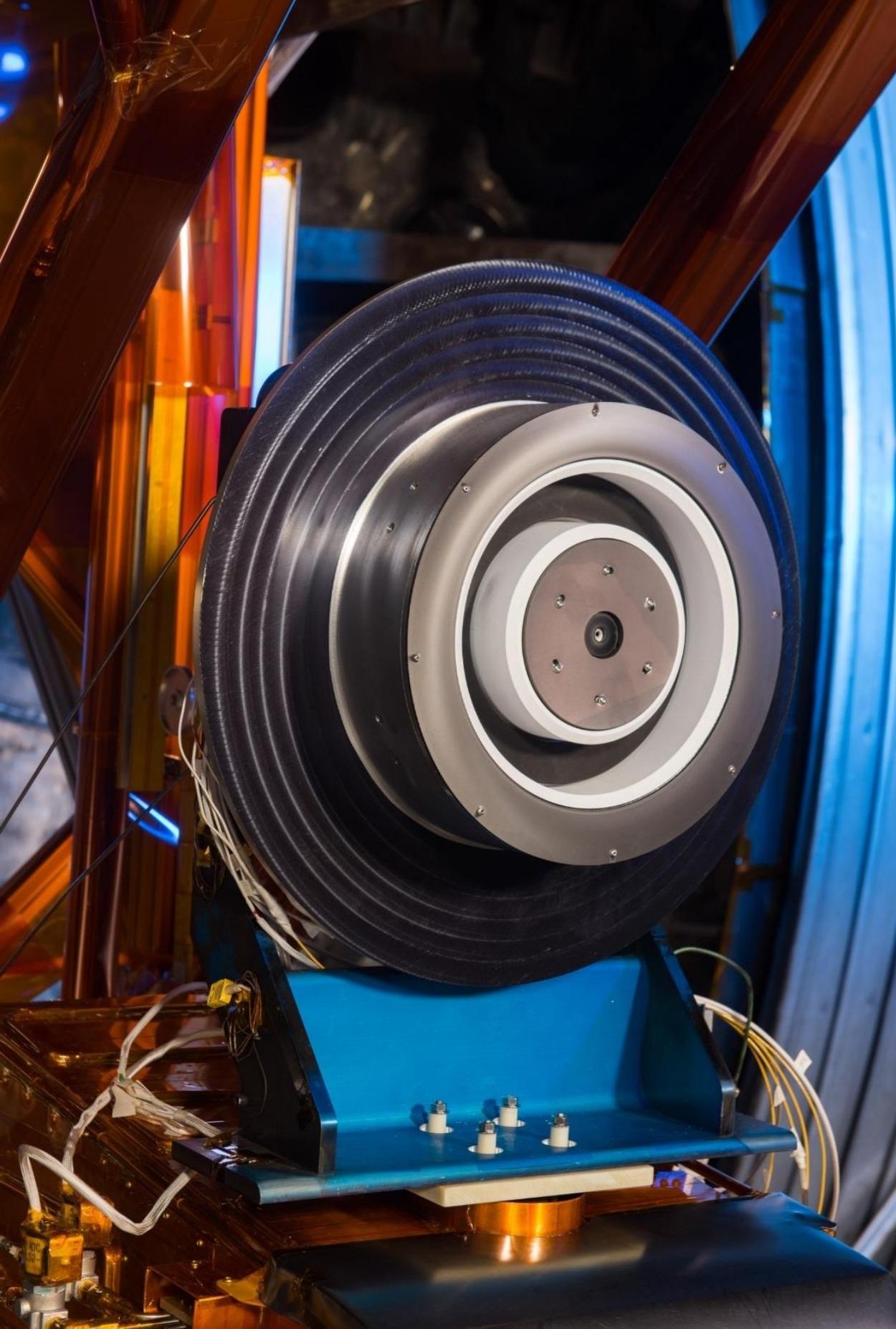

\title{
Uncertainty in Inverted Pendulum Thrust Measurements
}

J. Mackey, T. Haag, and H. Kamhawi NASA Glenn Research Center, Cleveland, Ohio 44135

S. Hall and P. Peterson

Vantage Partners LLC, Brook Park, Ohio 44142

54 ${ }^{\text {th }}$ AIAA/SAE/ASEE Joint Propulsion Conference 9 July 2018, Cincinnati, Ohio 


\section{Introduction}

- Thrust measurement is critical to the characterization of electric propulsion (EP) systems.

- Quantify: thrust, specific impulse, and efficiency.

- Challenging because of low thrust to weight ratios of EP systems.

- Sources of potential error must be well understood for reliable data.

- Strong need for detailed uncertainty analysis.

- NASA GRC vacuum facility 5 (VF-5) and vacuum facility 6 (VF-6) have similar inverted pendulum thrust stands.

- Several other NASA GRC thrust stands also exist with similar characteristics.
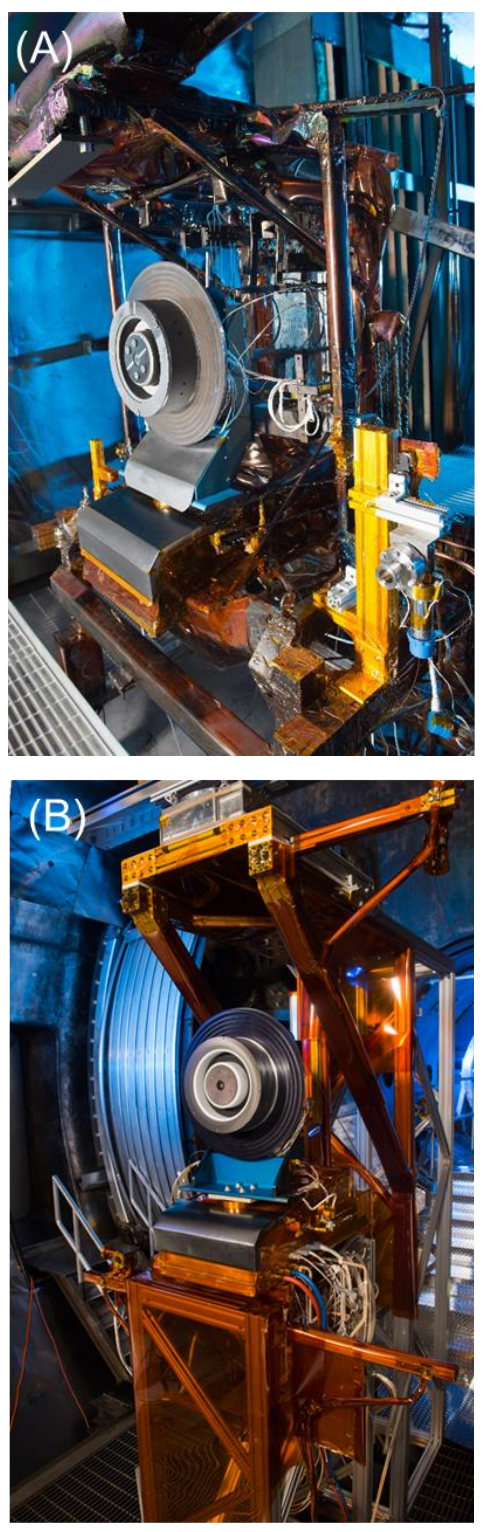


\section{Model of an Inverted Pendulum Stand}

Calibration Mode

a)

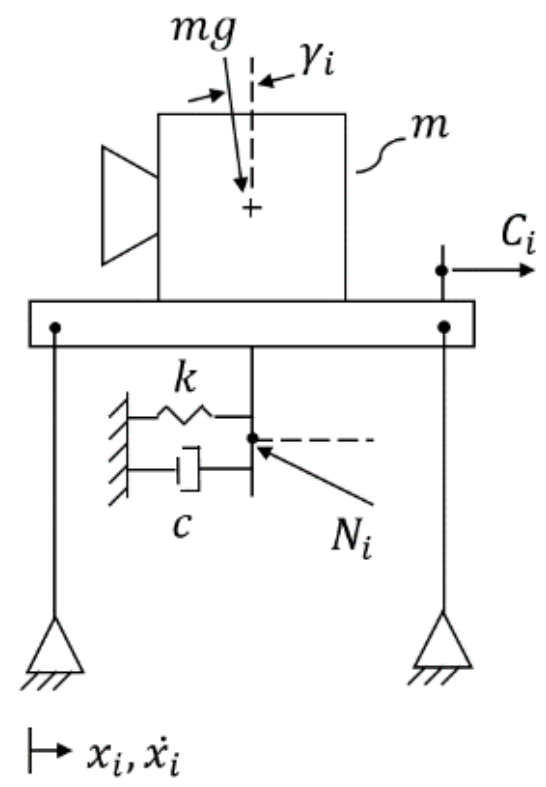

Calibration Details

b)

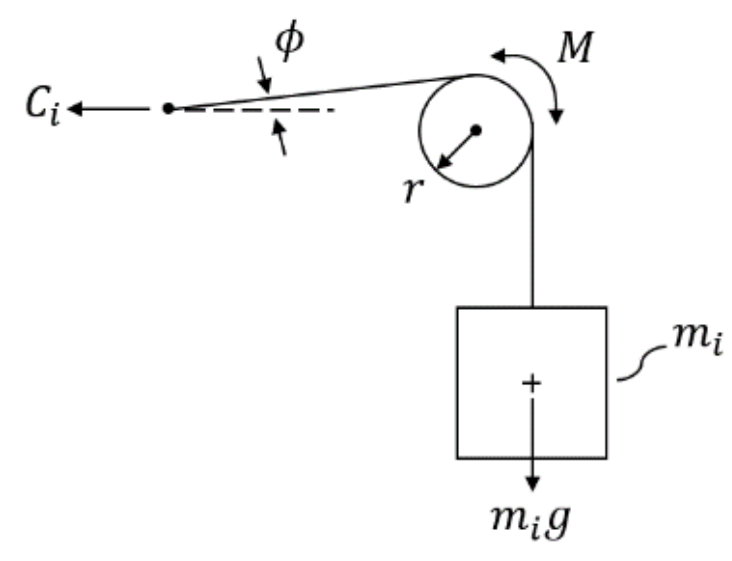

Thruster Mode

c)

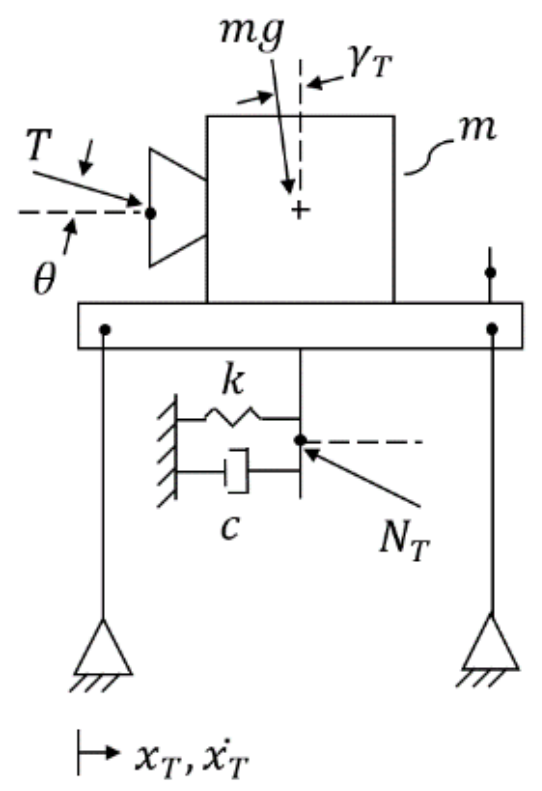




\section{Methodology}

- Classic approach of Figliola and Beasley, Abernethy et al., or Moffat.

- Design-stage, single-measurement

- Propagate error of independent sources using a truncated Taylor Series expansion.

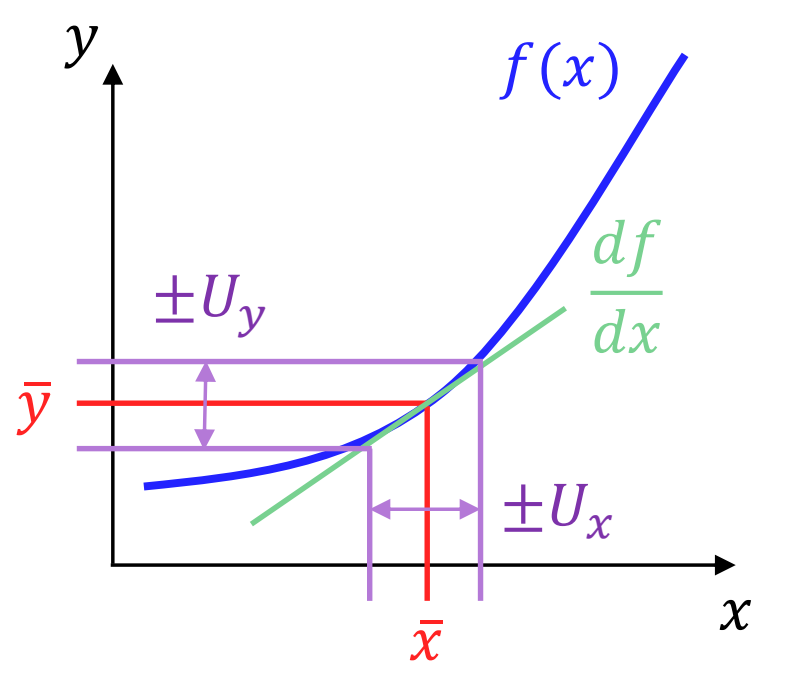

- Combine normalized error sources using a root sum of squares (RSS) type norm.

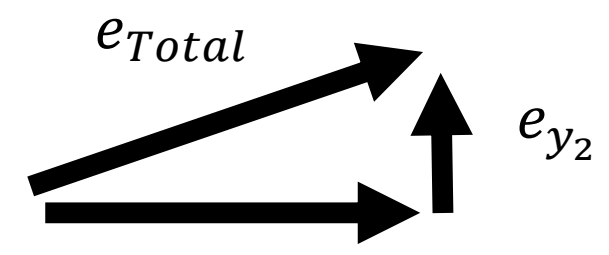

$e_{y_{1}}$

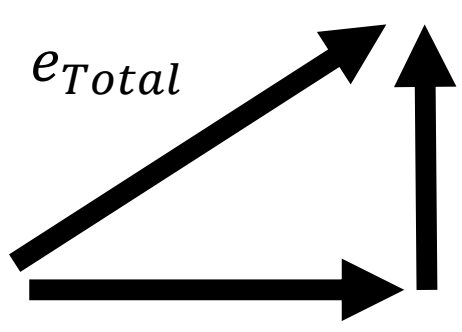

$e_{y_{1}}$

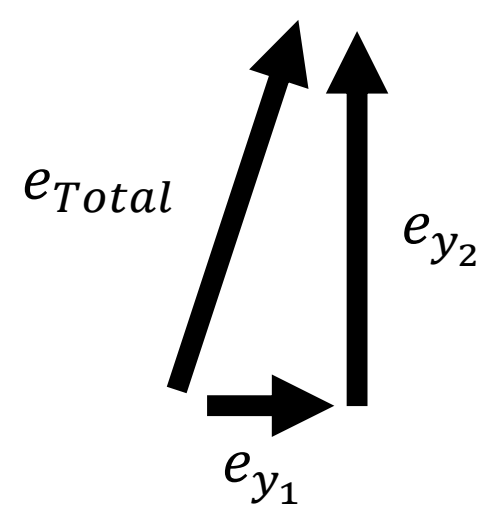




\section{Methodology (cont.)}

- Thrust stands can be operated in one of two modes:

- Displacement mode

" Load vs. deflected position

- Null coil mode

» Position held constant with a restoring force provided by a "null coil"

» Load vs. null coil current

- Example calibration dataset (right) can be reliably fit with a linear regression.

- Thrust can be estimated from calibration regression parameters and null coil shunt voltage.

- Objective of this work is to quantify the possible difference between a

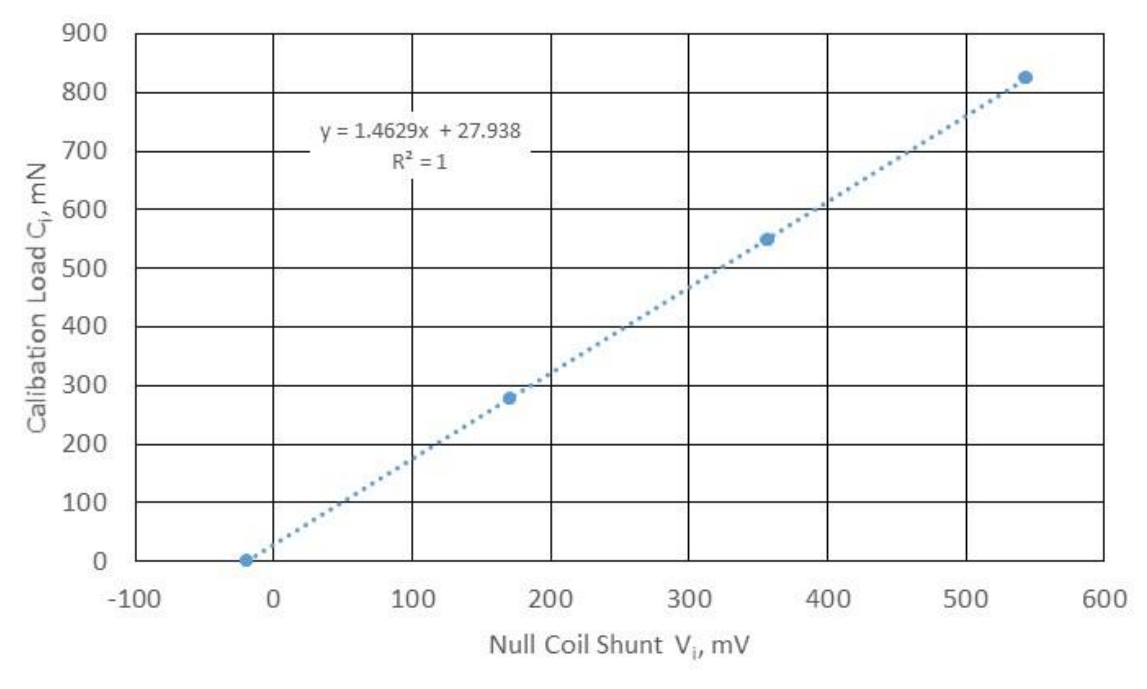
"measured" thrust and the "actual" thrust to some confidence level.

$$
T=a+b V_{T} \pm U_{T}
$$

$U_{T} 95 \%$ Confidence 


\section{Sources of Uncertainty}

\begin{tabular}{|c|c|c|c|}
\hline Source & Relative Uncertainty & $\begin{array}{c}\text { Parameters } \\
\text { of Interest }\end{array}$ & Parameter Description \\
\hline Thrust vector & $e_{\theta}=1-\cos (\theta)$ & $\theta$ & Thrust vector angle \\
\hline Stand displacement drift & $e_{x}=\frac{k\left|x_{T}-\tilde{x_{i}}\right|}{\bar{T}}$ & $\begin{array}{l}\left|x_{T}-\tilde{x}_{i}\right| \\
k=\omega_{n}^{2} m\end{array}$ & $\begin{array}{l}\text { Stand position drift, } \\
\text { Stiffness }\end{array}$ \\
\hline Stand velocity drift & $e_{\dot{x}}=\frac{c\left|\dot{x}_{T}-\tilde{\dot{x}}_{i}\right|}{\bar{T}}$ & $\begin{array}{c}\left|\dot{x}_{T}-\tilde{\dot{x}}_{i}\right| \\
c\end{array}$ & $\begin{array}{l}\text { Stand velocity drift, } \\
\text { Damping coefficient }\end{array}$ \\
\hline Stand inclination drift & $e_{\gamma}=\frac{m g \sin \left|\gamma_{T}-\widetilde{\gamma_{i}}\right|}{\bar{T}}$ & $\begin{array}{l}\left|\gamma_{T}-\widetilde{\gamma}_{i}\right| \\
m g\end{array}$ & $\begin{array}{l}\text { Stand inclination drift, } \\
\text { Thruster weight }\end{array}$ \\
\hline Shunt thermal drift & $e_{\text {shunt }}=\frac{\alpha\left|t_{T}-\widetilde{t_{i}}\right|}{\bar{R}_{\text {shunt }}}$ & $\begin{array}{c}\left|t_{T}-{\widetilde{t_{i}}}\right| \\
\quad \alpha \\
\bar{R}_{\text {shunt }}\end{array}$ & $\begin{array}{l}\text { Temperature drift, } \\
\text { Thermal sensitivity, } \\
\text { Nominal resistance }\end{array}$ \\
\hline $\begin{array}{l}\text { Calibration slope } \\
\text { repeatability }\end{array}$ & $e_{\text {slope }}=\frac{S_{b} \overline{V_{T}}}{\bar{T}}=S_{b}\left(\frac{1}{b}-\frac{a}{b \bar{T}}\right)$ & $S_{b}$ & $\begin{array}{l}\text { Calibration gain } \\
\text { standard deviation }\end{array}$ \\
\hline $\begin{array}{l}\text { Calibration regression } \\
\text { correlation }\end{array}$ & $e_{S_{x y}}=\frac{\sqrt{\frac{\sum\left(C_{i}-\left[a+b V_{i}\right]\right)^{2}}{n-2}}}{\bar{T}}$ & $\begin{array}{c}C_{i} \\
a+b V_{i}\end{array}$ & $\begin{array}{l}\text { Calibration force, } \\
\text { Calibration regression }\end{array}$ \\
\hline DAQ uncertainty & $e_{V_{i}}=\frac{U_{V_{i}}}{\overline{V_{T}}}=U_{V_{i}} \frac{\bar{T}-a}{b}$ & $U_{V_{i}}$ & $\begin{array}{l}\text { Data acquisition } \\
\text { uncertainty }\end{array}$ \\
\hline Calibration uncertainty & $e_{C_{i}}=\frac{1}{\bar{T}} \frac{\partial T}{\partial C_{i}} U_{C_{i}}$ & $U_{C_{i}}$ & $\begin{array}{l}\text { Calibration uncertainty, } \\
\text { see table } 2\end{array}$ \\
\hline
\end{tabular}




\section{Sources of Uncertainty}

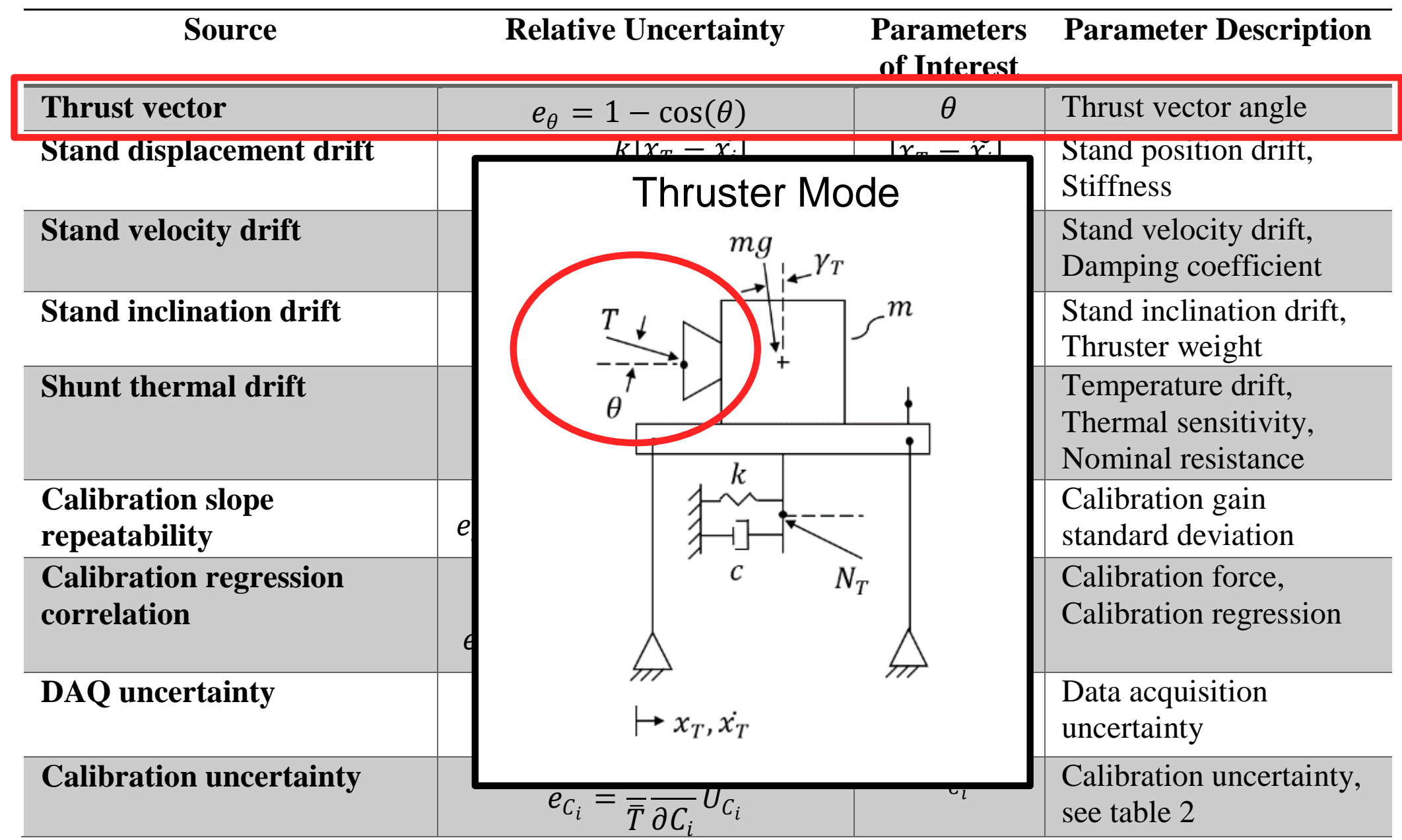




\section{Sources of Uncertainty}

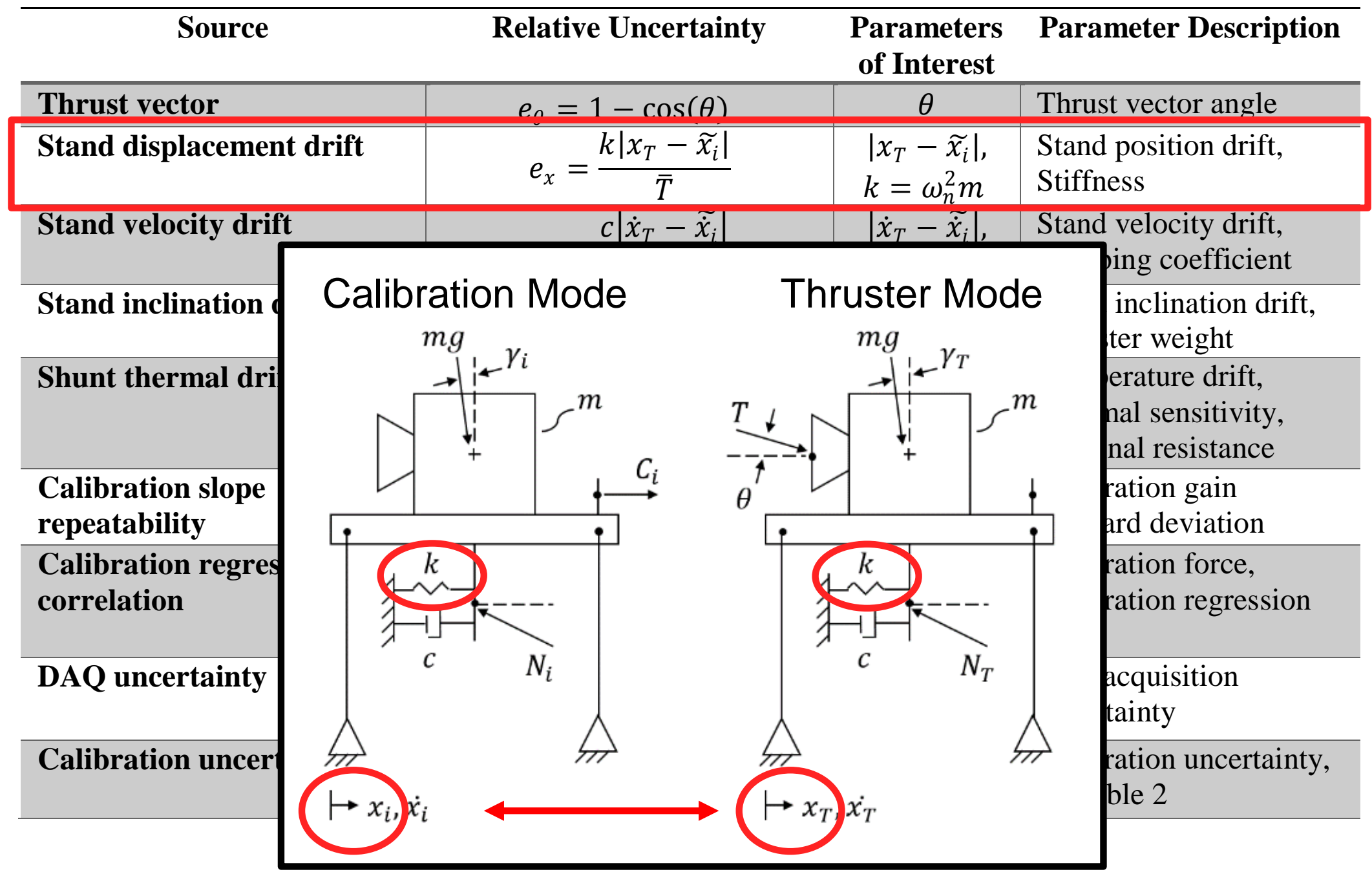




\section{Sources of Uncertainty}

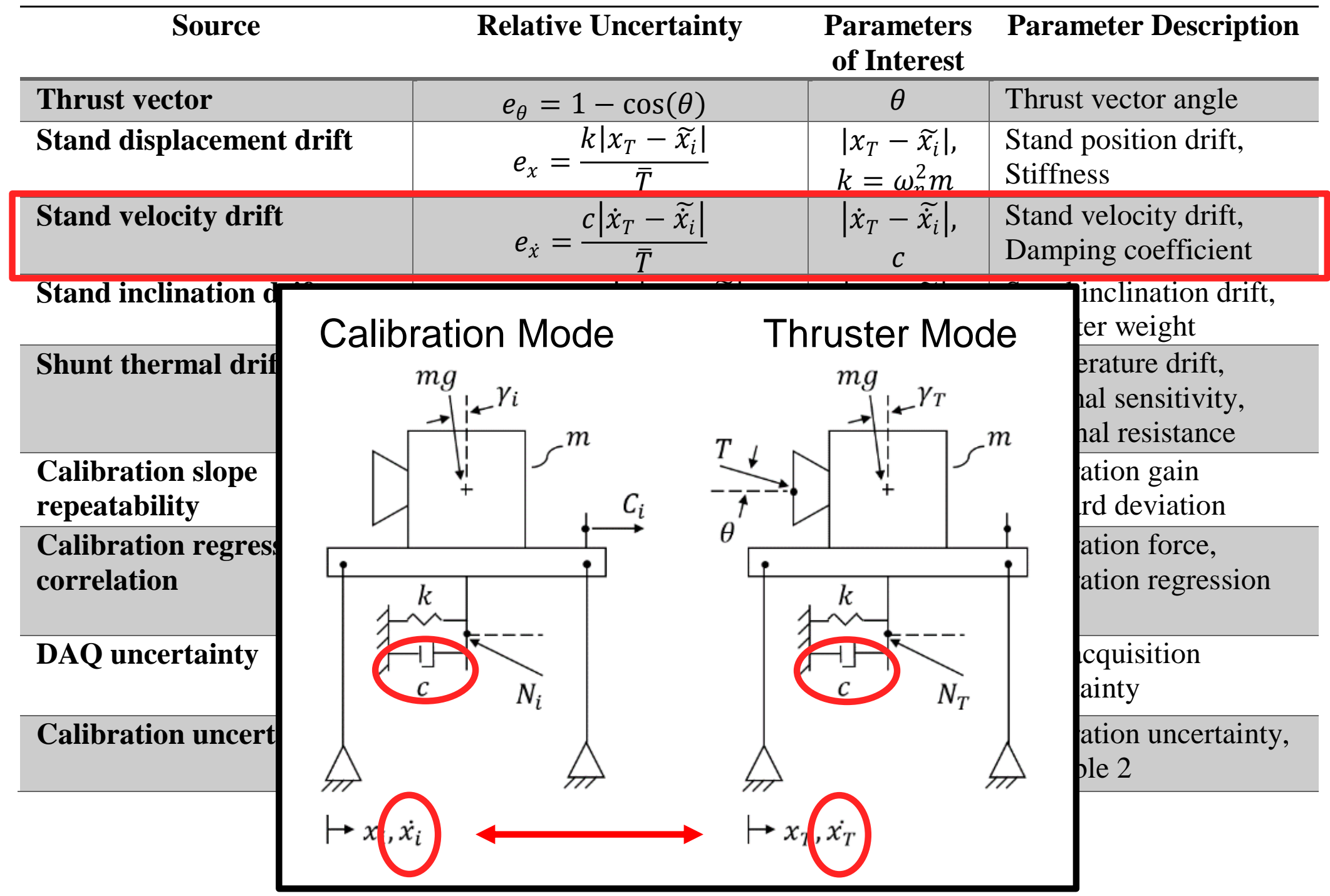




\section{Sources of Uncertainty}

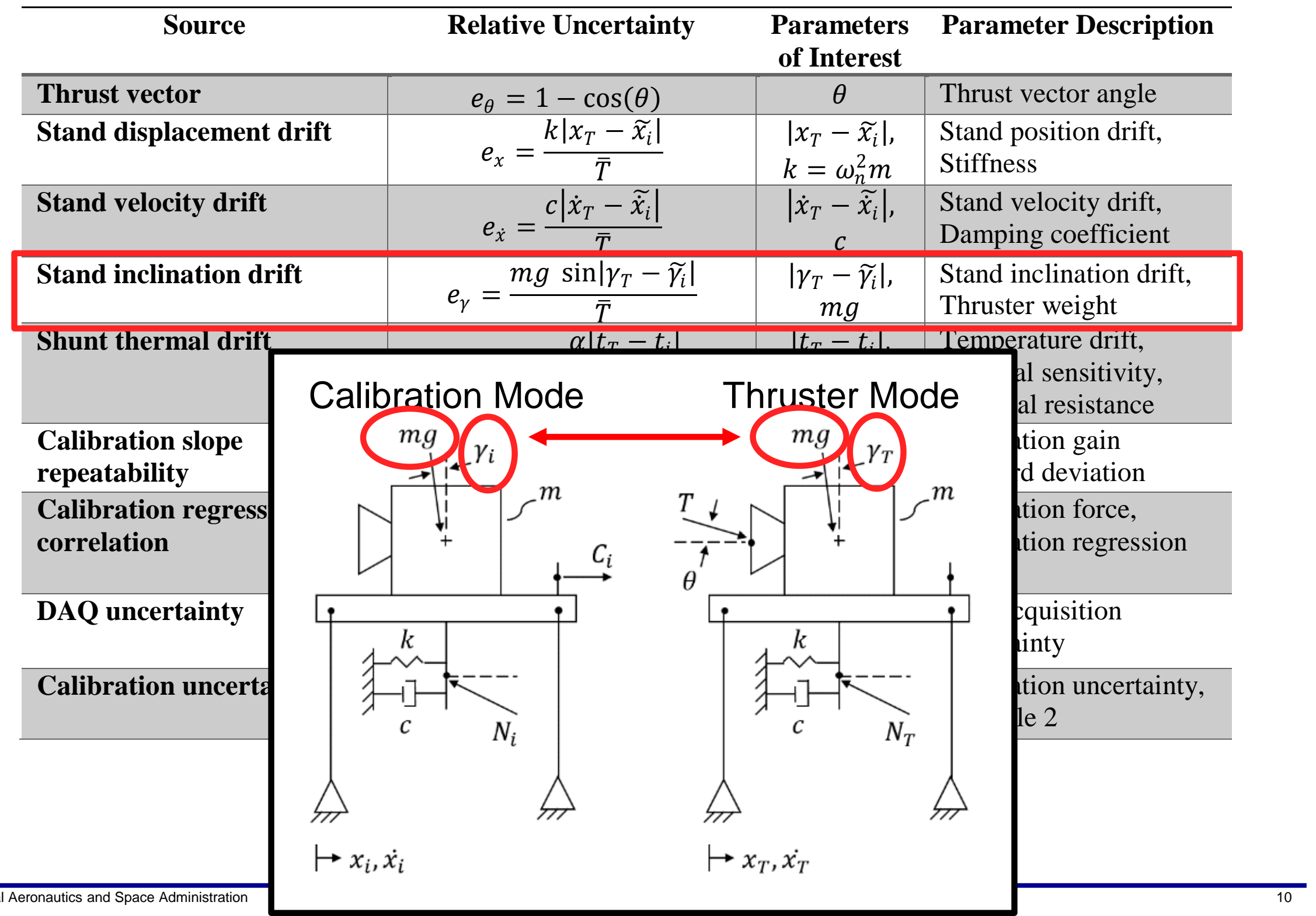




\section{Sources of Uncertainty}

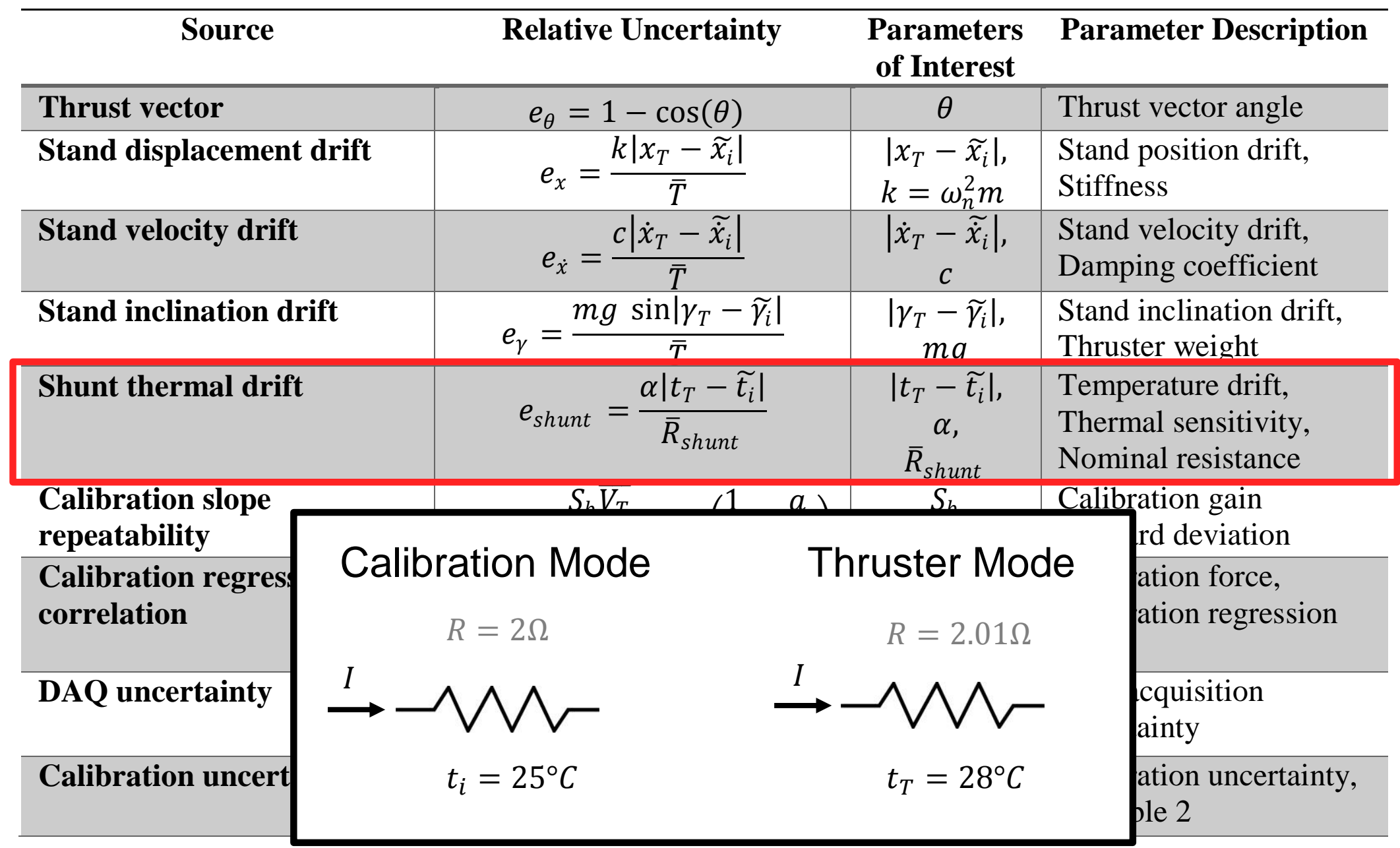




\section{Sources of Uncertainty}

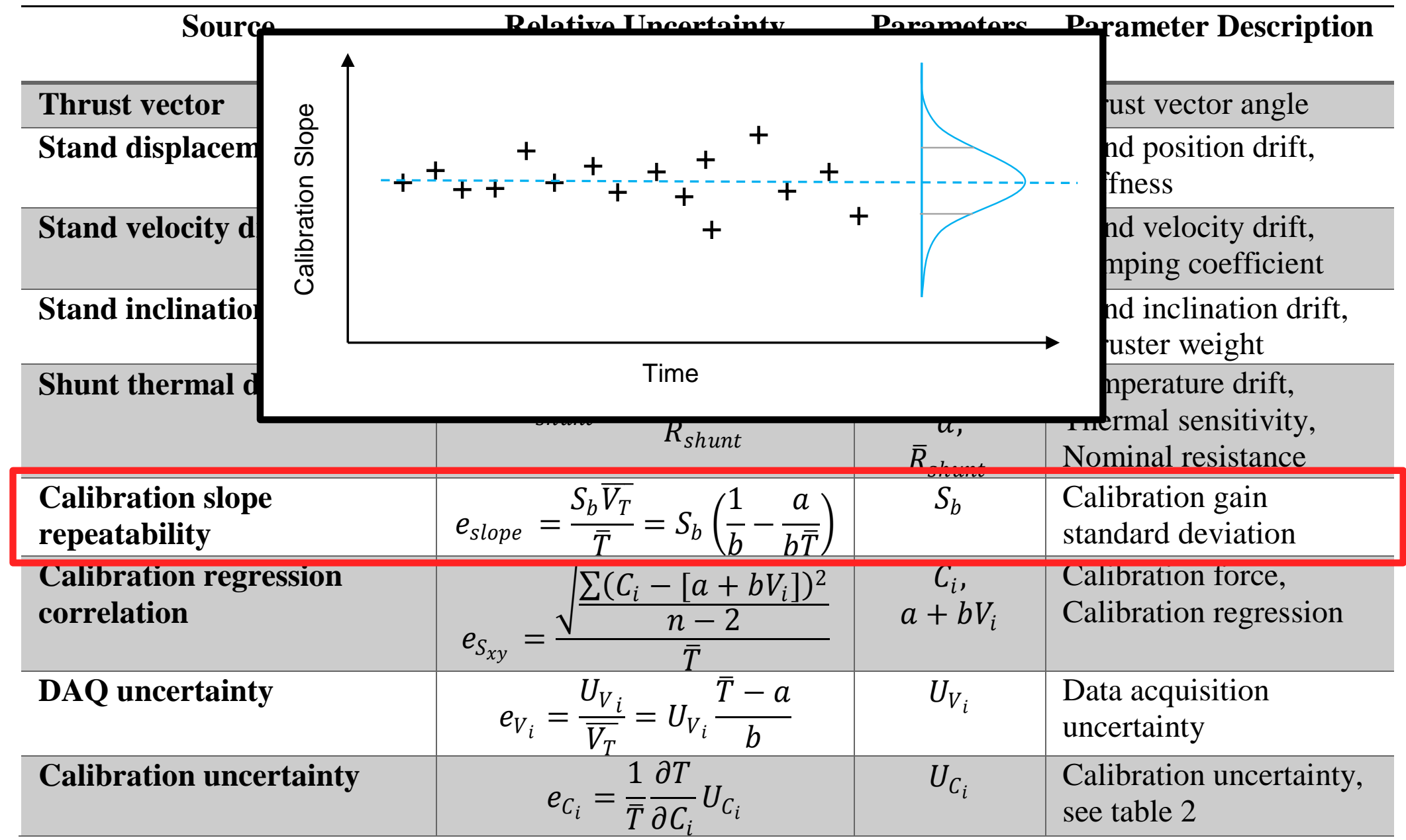




\section{Sources of Uncertainty}

\begin{tabular}{|c|c|c|c|}
\hline Source & Relative Uncertainty & $\begin{array}{c}\text { Parameters } \\
\text { of Interest }\end{array}$ & Parameter Description \\
\hline Thrust vector & $e_{\theta}=1-\cos (\theta)$ & $\theta$ & Thrust vector angle \\
\hline Stand displacement drift & & i, & $\begin{array}{l}\text { Stand position drift, } \\
\text { Stiffness }\end{array}$ \\
\hline Stand velocity drift & 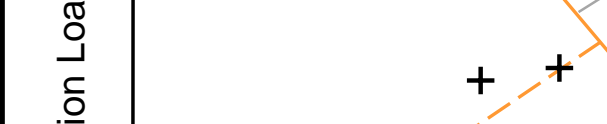 & & $\begin{array}{l}\text { Stand velocity drift, } \\
\text { Damping coefficient }\end{array}$ \\
\hline Stand inclination drift & $\frac{\bar{\pi}}{\frac{\pi}{0}}$ & l, & $\begin{array}{l}\text { Stand inclination drift, } \\
\text { Thruster weight }\end{array}$ \\
\hline Shunt thermal drift & Shunt Voltage & l, & $\begin{array}{l}\text { Temperature drift, } \\
\text { Thermal sensitivity, } \\
\text { Nominal resistance }\end{array}$ \\
\hline $\begin{array}{l}\text { Calibration slope } \\
\text { repeatability }\end{array}$ & $e_{\text {slope }}=\frac{J_{b} v_{T}}{\bar{T}}=S_{b}\left(\frac{1}{b}-\frac{a}{h \bar{T}}\right)$ & $\sigma_{b}$ & $\begin{array}{l}\text { Calibration gain } \\
\text { standard deviation }\end{array}$ \\
\hline $\begin{array}{l}\text { Calibration regression } \\
\text { correlation }\end{array}$ & $e_{S_{x y}}=\frac{\sqrt{\frac{\sum\left(C_{i}-\left[a+b V_{i}\right]\right)^{2}}{n-2}}}{\bar{T}}$ & $\begin{array}{c}C_{i} \\
a+b V_{i}\end{array}$ & $\begin{array}{l}\text { Calibration force, } \\
\text { Calibration regression }\end{array}$ \\
\hline DAQ uncertainty & $e_{V_{i}}=\frac{U_{V_{i}}}{\overline{V_{T}}}=U_{V_{i}} \frac{T-a}{b}$ & $U_{V_{i}}$ & $\begin{array}{l}\text { Data acquisition } \\
\text { uncertainty }\end{array}$ \\
\hline Calibration uncertainty & $e_{C_{i}}=\frac{1}{\bar{T}} \frac{\partial T}{\partial C_{i}} U_{C_{i}}$ & $U_{C_{i}}$ & $\begin{array}{l}\text { Calibration uncertainty, } \\
\text { see table } 2\end{array}$ \\
\hline
\end{tabular}




\section{Sources of Uncertainty}

\begin{tabular}{|c|c|c|c|}
\hline Source & Relative Uncertainty & $\begin{array}{c}\text { Parameters } \\
\text { of Interest }\end{array}$ & Parameter Description \\
\hline Thrust vector & $e_{\theta}=1-\cos (\theta)$ & $\theta$ & Thrust vector angle \\
\hline Stand displacement drift & & il & $\begin{array}{l}\text { Stand position drift, } \\
\text { Stiffness }\end{array}$ \\
\hline Stand velocity drift & 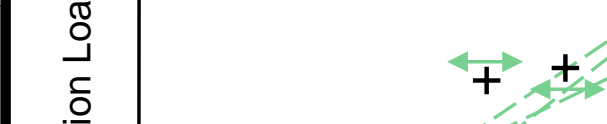 & , & $\begin{array}{l}\text { Stand velocity drift, } \\
\text { Damping coefficient }\end{array}$ \\
\hline Stand inclination drift & 产 & l, & $\begin{array}{l}\text { Stand inclination drift, } \\
\text { Thruster weight }\end{array}$ \\
\hline Shunt thermal drift & Shunt Voltage & |, & $\begin{array}{l}\text { Temperature drift, } \\
\text { Thermal sensitivity, } \\
\text { Nominal resistance }\end{array}$ \\
\hline $\begin{array}{l}\text { Calibration slope } \\
\text { repeatability }\end{array}$ & $e_{\text {slope }}=\frac{s_{b} v_{T}}{\bar{T}}=S_{b}\left(\frac{1}{b}-\frac{a}{b \bar{T}}\right)$ & $\boldsymbol{J}_{b}$ & $\begin{array}{l}\text { Calibration gain } \\
\text { standard deviation }\end{array}$ \\
\hline $\begin{array}{l}\text { Calibration regression } \\
\text { correlation }\end{array}$ & $e_{S_{x y}}=\frac{\sqrt{\frac{\sum\left(C_{i}-\left[a+b V_{i}\right]\right)^{2}}{n-2}}}{\overline{\boldsymbol{T}}}$ & $\begin{array}{c}C_{i} \\
a+b V_{i}\end{array}$ & $\begin{array}{l}\text { Calibration force, } \\
\text { Calibration regression }\end{array}$ \\
\hline DAQ uncertainty & $e_{V_{i}}=\frac{U_{V_{i}}}{\overline{V_{T}}}=U_{V_{i}} \frac{\bar{T}-a}{b}$ & $U_{V_{i}}$ & $\begin{array}{l}\text { Data acquisition } \\
\text { uncertainty }\end{array}$ \\
\hline Calibration uncertainty & $e_{C_{i}}=\frac{1}{\bar{T}} \frac{\partial T}{\partial C_{i}} U_{C_{i}}$ & $U_{C_{i}}$ & $\begin{array}{l}\text { Calibration uncertainty, } \\
\text { see table } 2\end{array}$ \\
\hline
\end{tabular}




\section{Sources of Uncertainty}

\begin{tabular}{|c|c|c|c|}
\hline Source & Relative Uncertainty & $\begin{array}{c}\text { Parameters } \\
\text { of Interest }\end{array}$ & Parameter Description \\
\hline Thrust vector & $e_{\theta}=1-\cos (\theta)$ & $\theta$ & Thrust vector angle \\
\hline Stand displacement drift & & i, & $\begin{array}{l}\text { Stand position drift, } \\
\text { Stiffness }\end{array}$ \\
\hline Stand velocity drift & 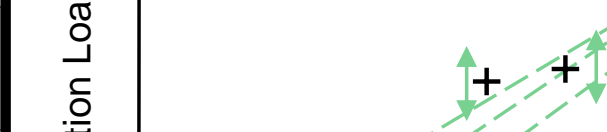 & , & $\begin{array}{l}\text { Stand velocity drift, } \\
\text { Damping coefficient }\end{array}$ \\
\hline Stand inclination drift & $\frac{\sqrt{0}}{\bar{\sigma}}$ & t, & $\begin{array}{l}\text { Stand inclination drift, } \\
\text { Thruster weight }\end{array}$ \\
\hline Shunt thermal drift & Shunt Voltage & |, & $\begin{array}{l}\text { Temperature drift, } \\
\text { Thermal sensitivity, } \\
\text { Nominal resistance }\end{array}$ \\
\hline $\begin{array}{l}\text { Calibration slope } \\
\text { repeatability }\end{array}$ & $e_{\text {slope }}=\frac{s_{b^{v}}}{\bar{T}}=S_{b}\left(\frac{1}{b}-\frac{a}{b \bar{T}}\right)$ & $\tau_{b}$ & $\begin{array}{l}\text { Calibration gain } \\
\text { standard deviation }\end{array}$ \\
\hline $\begin{array}{l}\text { Calibration regression } \\
\text { correlation }\end{array}$ & $e_{S_{x y}}=\frac{\sqrt{\frac{\sum\left(C_{i}-\left[a+b V_{i}\right]\right)^{2}}{n-2}}}{\bar{T}}$ & $\begin{array}{c}C_{i} \\
a+b V_{i}\end{array}$ & $\begin{array}{l}\text { Calibration force, } \\
\text { Calibration regression }\end{array}$ \\
\hline DAQ uncertainty & $e_{V_{i}}=\frac{U_{V_{i}}}{\overline{V_{T}}}=U_{V_{i}} \frac{\bar{T}-a}{b}$ & $U_{V_{i}}$ & $\begin{array}{l}\text { Data acquisition } \\
\text { uncertainty }\end{array}$ \\
\hline Calibration uncertainty & $e_{C_{i}}=\frac{1}{\bar{T}} \frac{\partial T}{\partial C_{i}} U_{C_{i}}$ & $U_{C_{i}}$ & $\begin{array}{l}\text { Calibration uncertainty, } \\
\text { see table } 2\end{array}$ \\
\hline
\end{tabular}




\section{Calibration Sources of Uncertainty}

\begin{tabular}{|c|c|c|c|}
\hline Source & Relative Uncertainty & $\begin{array}{c}\text { Parameters } \\
\text { of Interest }\end{array}$ & Parameter Description \\
\hline Calibration alignment & $e_{\varphi}=1-\cos (\varphi)$ & $\varphi$ & $\begin{array}{l}\text { Calibration alignment } \\
\text { angle }\end{array}$ \\
\hline Calibration pulley moment & $e_{M}=\frac{M}{r \bar{C}_{i}}$ & $M$ & $\begin{array}{l}\text { Calibration pulley } \\
\text { moment }\end{array}$ \\
\hline $\begin{array}{l}\text { Calibration mass } \\
\text { uncertainty }\end{array}$ & $e_{m_{i}}=\frac{U_{m_{i}} g}{\bar{C}_{i}}$ & $U_{m_{i}}$ & $\begin{array}{l}\text { Calibration mass } \\
\text { uncertainty }\end{array}$ \\
\hline $\begin{array}{l}\text { Calibration gravity } \\
\text { uncertainty }\end{array}$ & $e_{g}=\frac{U_{g}}{g}$ & $U_{g}$ & $\begin{array}{l}\text { Calibration gravity } \\
\text { uncertainty }\end{array}$ \\
\hline
\end{tabular}




\section{Calibration Sources of Uncertainty}

\begin{tabular}{|c|c|c|c|}
\hline Source & Relative Uncertainty & $\begin{array}{c}\text { Parameters } \\
\text { of Interest }\end{array}$ & Parameter Description \\
\hline Calibration alignment & $e_{\varphi}=1-\cos (\varphi)$ & $\varphi$ & $\begin{array}{l}\text { Calibration alignment } \\
\text { angle }\end{array}$ \\
\hline Calibration pulley moment & $e_{M}=\frac{M}{r \overline{C_{i}}}$ & $M$ & $\begin{array}{l}\text { Calibration pulley } \\
\text { moment }\end{array}$ \\
\hline $\begin{array}{l}\text { Calibration mass } \\
\text { uncertainty }\end{array}$ & $e_{m_{i}}=\frac{U_{m_{i}} g}{\bar{C}_{i}}$ & $U_{m_{i}}$ & $\begin{array}{l}\text { Calibration mass } \\
\text { uncertainty }\end{array}$ \\
\hline $\begin{array}{l}\text { Calibration gravity } \\
\text { uncertainty }\end{array}$ & $e_{g}=\frac{U_{g}}{g}$ & $U_{g}$ & $\begin{array}{l}\text { Calibration gravity } \\
\text { uncertainty }\end{array}$ \\
\hline
\end{tabular}

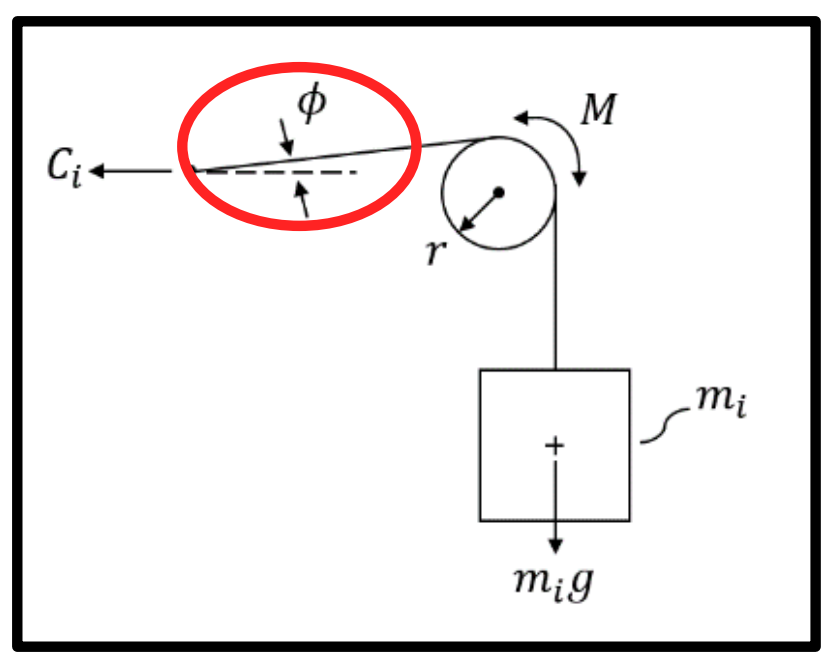




\section{Calibration Sources of Uncertainty}

\begin{tabular}{|c|c|c|c|}
\hline Source & Relative Uncertainty & $\begin{array}{c}\text { Parameters } \\
\text { of Interest }\end{array}$ & Parameter Description \\
\hline Calibration alignment & $e_{\varphi}=1-\cos (\varphi)$ & $\varphi$ & $\begin{array}{l}\text { Calibration alignment } \\
\text { angle }\end{array}$ \\
\hline Calibration pulley moment & $e_{M}=\frac{M}{r \overline{C_{i}}}$ & $M$ & $\begin{array}{l}\text { Calibration pulley } \\
\text { moment }\end{array}$ \\
\hline $\begin{array}{l}\text { Calibration mass } \\
\text { uncertainty }\end{array}$ & $e_{m_{i}}=\frac{U_{m_{i}} g}{\bar{C}_{i}}$ & $U_{m_{i}}$ & $\begin{array}{l}\text { Calibration mass } \\
\text { uncertainty }\end{array}$ \\
\hline $\begin{array}{l}\text { Calibration gravity } \\
\text { uncertainty }\end{array}$ & $e_{g}=\frac{U_{g}}{g}$ & $U_{g}$ & $\begin{array}{l}\text { Calibration gravity } \\
\text { uncertainty }\end{array}$ \\
\hline
\end{tabular}

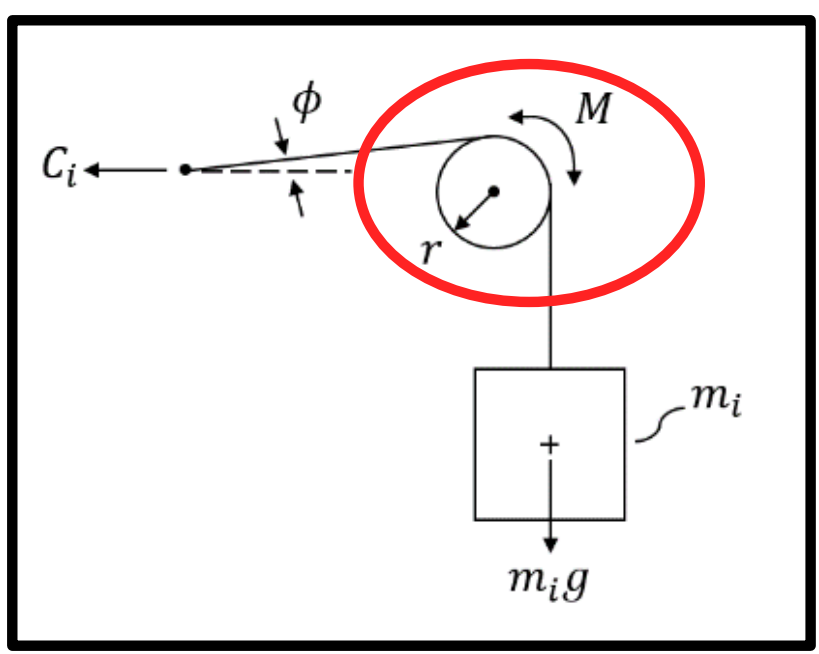




\section{Calibration Sources of Uncertainty}

\begin{tabular}{|c|c|c|c|}
\hline Source & Relative Uncertainty & $\begin{array}{c}\text { Parameters } \\
\text { of Interest }\end{array}$ & Parameter Description \\
\hline Calibration alignment & $e_{\varphi}=1-\cos (\varphi)$ & $\varphi$ & $\begin{array}{l}\text { Calibration alignment } \\
\text { angle }\end{array}$ \\
\hline Calibration pulley moment & $e_{M}=\frac{M}{r \bar{C}_{i}}$ & $M$ & $\begin{array}{l}\text { Calibration pulley } \\
\text { moment }\end{array}$ \\
\hline $\begin{array}{l}\text { Calibration mass } \\
\text { uncertainty }\end{array}$ & $e_{m_{i}}=\frac{U_{m_{i}} g}{\bar{C}_{i}}$ & $U_{m_{i}}$ & $\begin{array}{l}\text { Calibration mass } \\
\text { uncertainty }\end{array}$ \\
\hline $\begin{array}{l}\text { Calibration gravity } \\
\text { uncertainty }\end{array}$ & $e_{g}=\frac{U_{g}}{g}$ & $U_{g}$ & $\begin{array}{l}\text { Calibration gravity } \\
\text { uncertainty }\end{array}$ \\
\hline
\end{tabular}

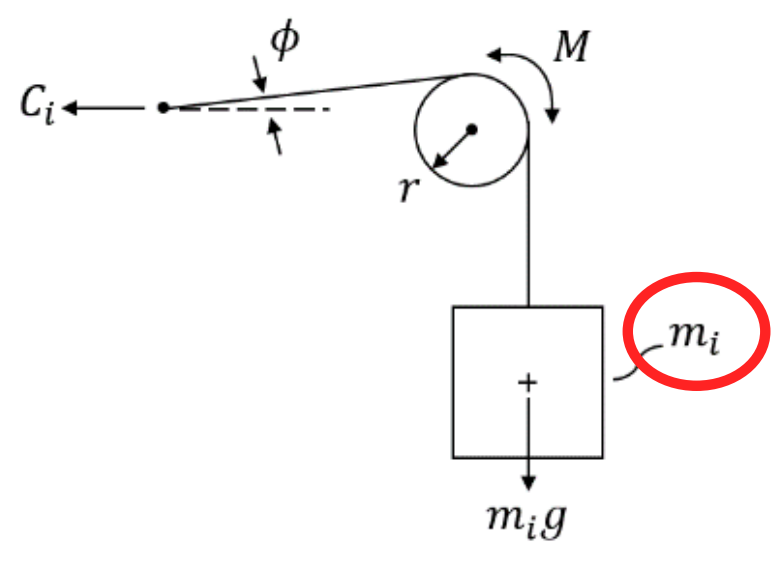




\section{Calibration Sources of Uncertainty}

\begin{tabular}{|c|c|c|c|}
\hline Source & Relative Uncertainty & $\begin{array}{c}\text { Parameters } \\
\text { of Interest }\end{array}$ & Parameter Description \\
\hline Calibration alignment & $e_{\varphi}=1-\cos (\varphi)$ & $\varphi$ & $\begin{array}{l}\text { Calibration alignment } \\
\text { angle }\end{array}$ \\
\hline Calibration pulley moment & $e_{M}=\frac{M}{r \bar{C}_{i}}$ & $M$ & $\begin{array}{l}\text { Calibration pulley } \\
\text { moment }\end{array}$ \\
\hline $\begin{array}{l}\text { Calibration mass } \\
\text { uncertainty }\end{array}$ & $e_{m_{i}}=\frac{U_{m_{i}} g}{\bar{C}_{i}}$ & $U_{m_{i}}$ & $\begin{array}{l}\text { Calibration mass } \\
\text { uncertainty }\end{array}$ \\
\hline $\begin{array}{l}\text { Calibration gravity } \\
\text { uncertainty }\end{array}$ & $e_{g}=\frac{U_{g}}{g}$ & $U_{g}$ & $\begin{array}{l}\text { Calibration gravity } \\
\text { uncertainty }\end{array}$ \\
\hline
\end{tabular}

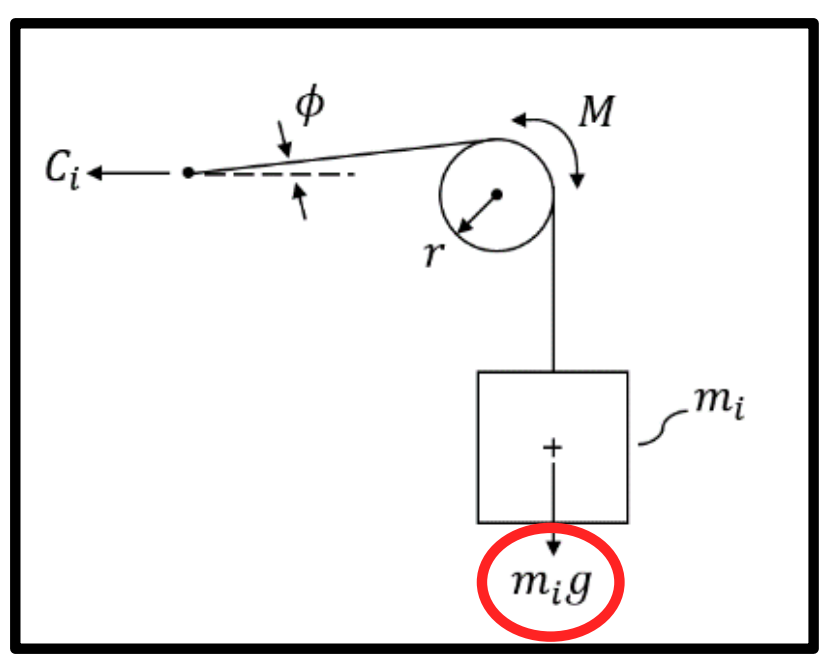




\section{Combination of Sources}

$$
\begin{gathered}
e_{T}=\frac{U_{T}}{\bar{T}}=\sqrt{e_{\theta}^{2}+e_{x}^{2}+e_{\grave{x}}^{2}+e_{\gamma}^{2}+e_{\text {Shunt }}^{2}+e_{\text {Slope }}^{2}+e_{S_{x y}}^{2}+e_{V_{i}}^{2} \sqrt{n}+e_{C_{i}}^{2} \sqrt{n}} . \\
U_{C_{i}}=\bar{T} \sqrt{e_{\varphi}^{2}+e_{M}^{2}+e_{m_{i}}^{2}+e_{g}^{2}}
\end{gathered}
$$

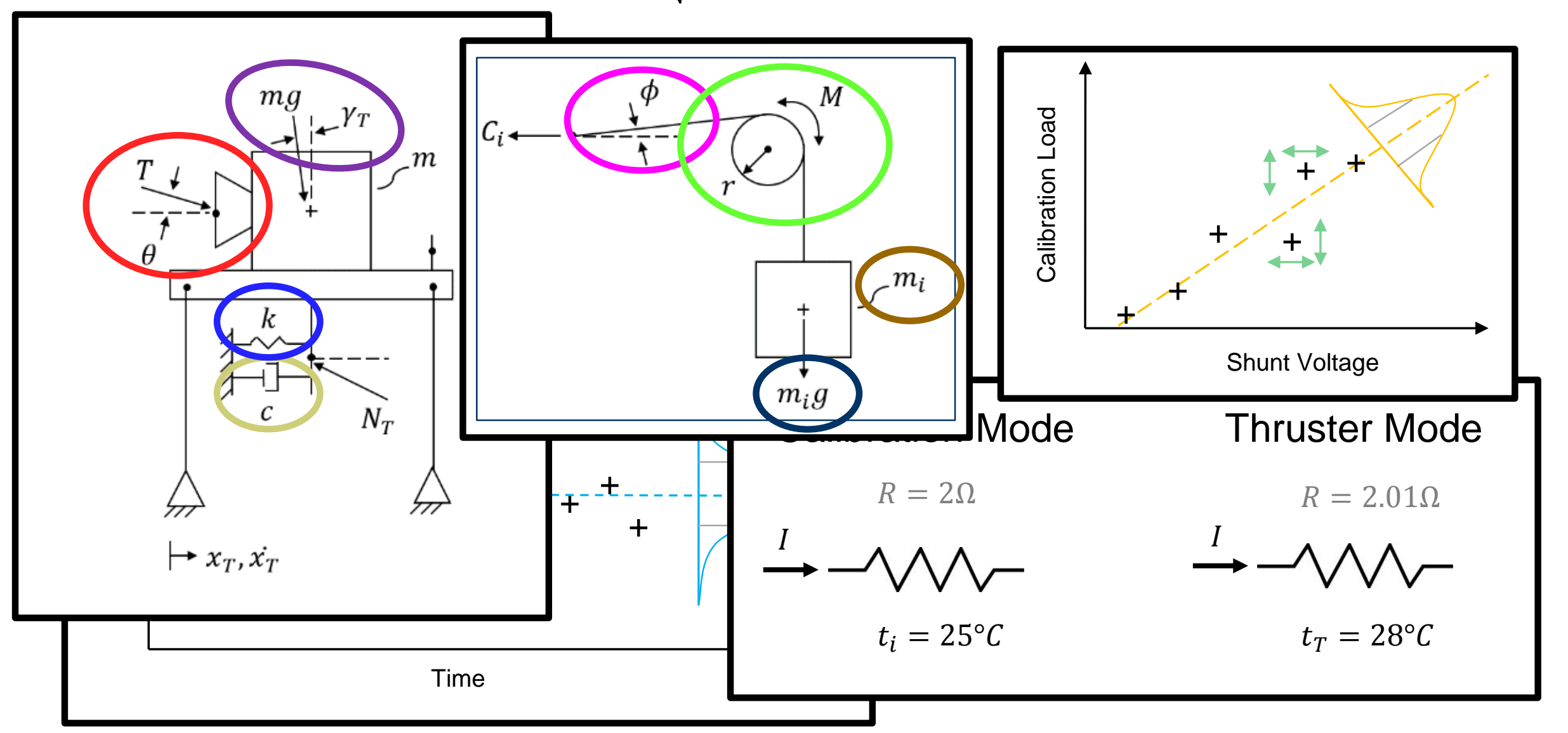




\section{Case Study NASA VF-6 Thrust Stand}

- VF-6 inverted pendulum thrust stand has been recently constructed with uncertainty quantification in mind.

- Technology Development Unit (TDU) 12.5 kW Hall thruster for Advanced Electric Propulsion System (AEPS).

- Null and damper coils on PID feedback loops.

- Inclination control on PID feedback loop.

- Linear variable differential transformer (LVDT) position measurement for PID feedback.

- Laser triangulation sensor as secondary position measurement.

- Electrolytic inclinometer tilt sensor for PID feedback.

- Inertial inclinometer tilt sensor for secondary inclination measurement.

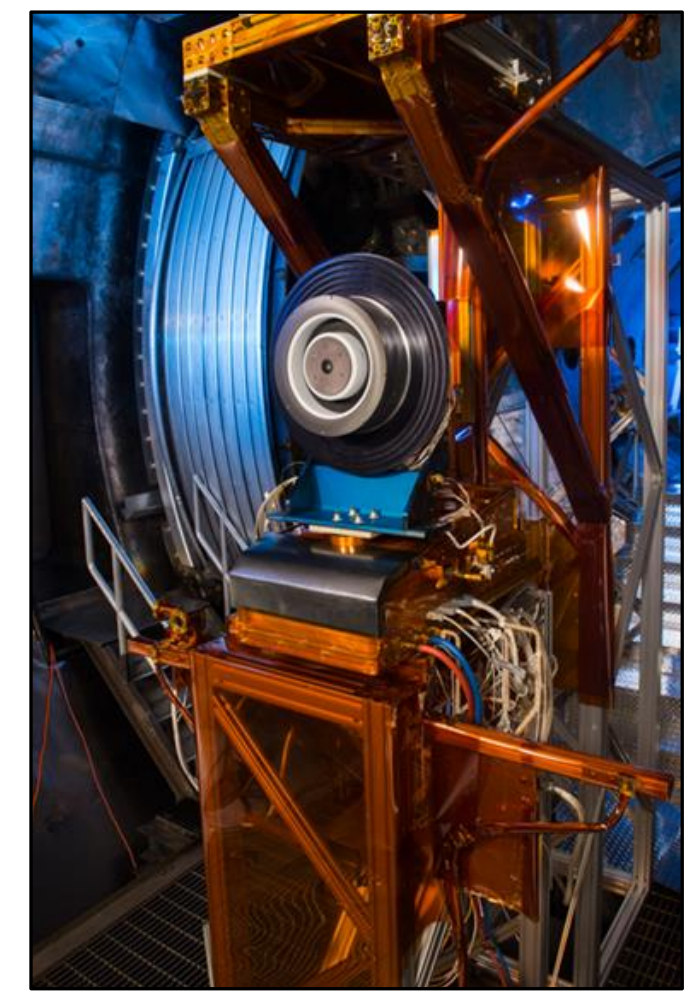

- In-situ calibration mechanism.

- Protective thermal/environmental shroud. 


\section{Stand Characterization}

- Stand stiffness, damping coefficient, and total dynamic mass were calculated.

- May change with different thrusters, different thrust stands, and different setup configurations.

Displacement Mode Operation
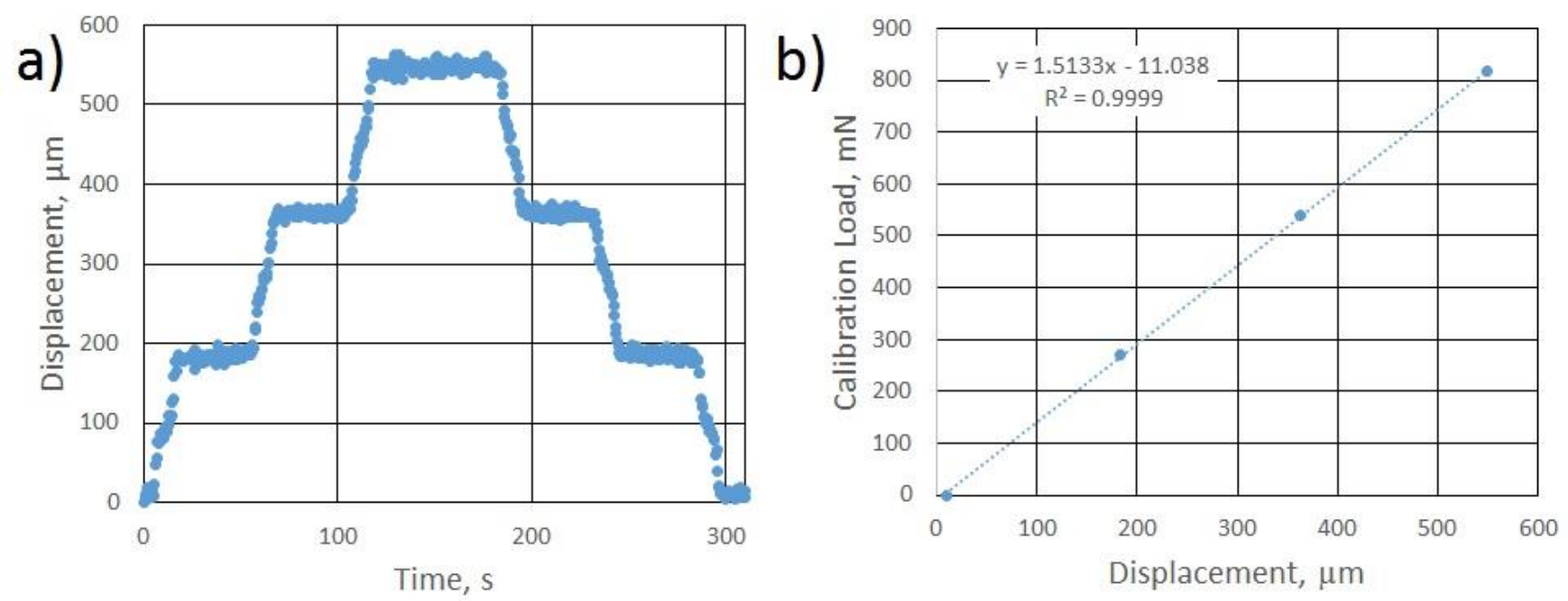

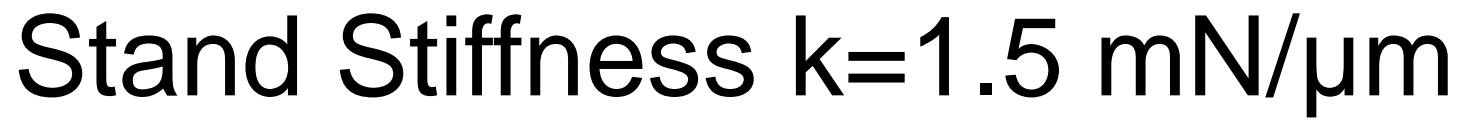




\section{Stand Characterization (cont.)}

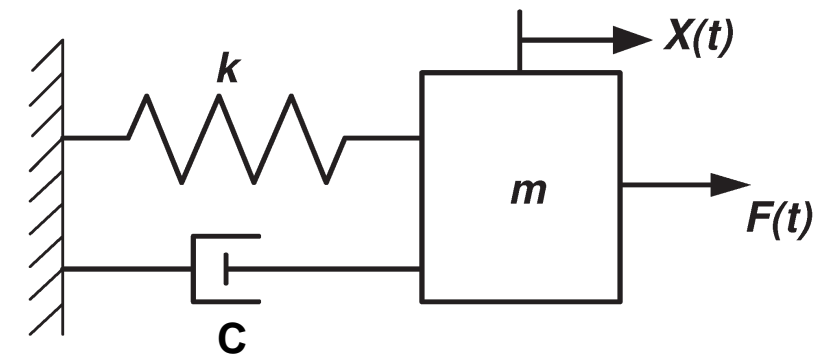

"Free" Response to Impulse, No Active Coils
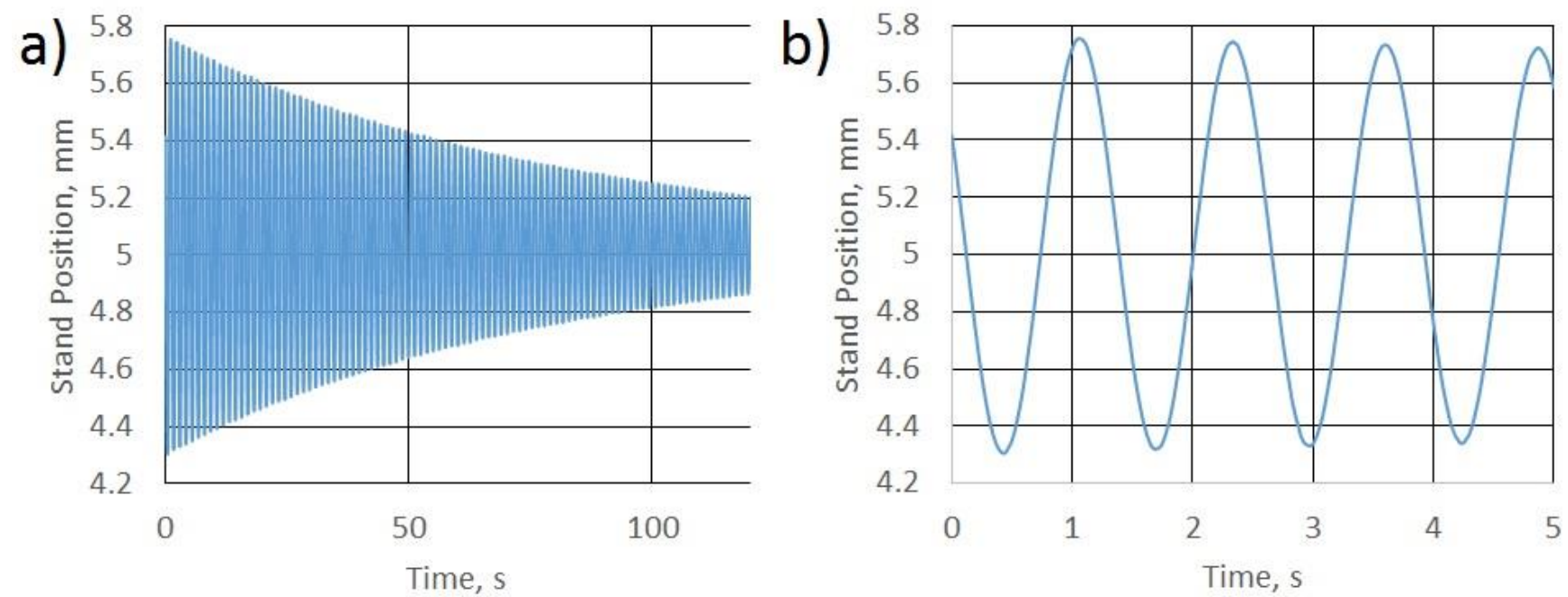

Stand Damping Coefficient $\mathrm{C}=1.5 \mathrm{~kg} / \mathrm{s}(\xi=2.5 \mathrm{e}-3)$ Stand Natural Frequency $\omega_{n}=0.788 \mathrm{~Hz}$ 


\section{Stand Characterization (cont.)}

Nominal Quiescent Operation

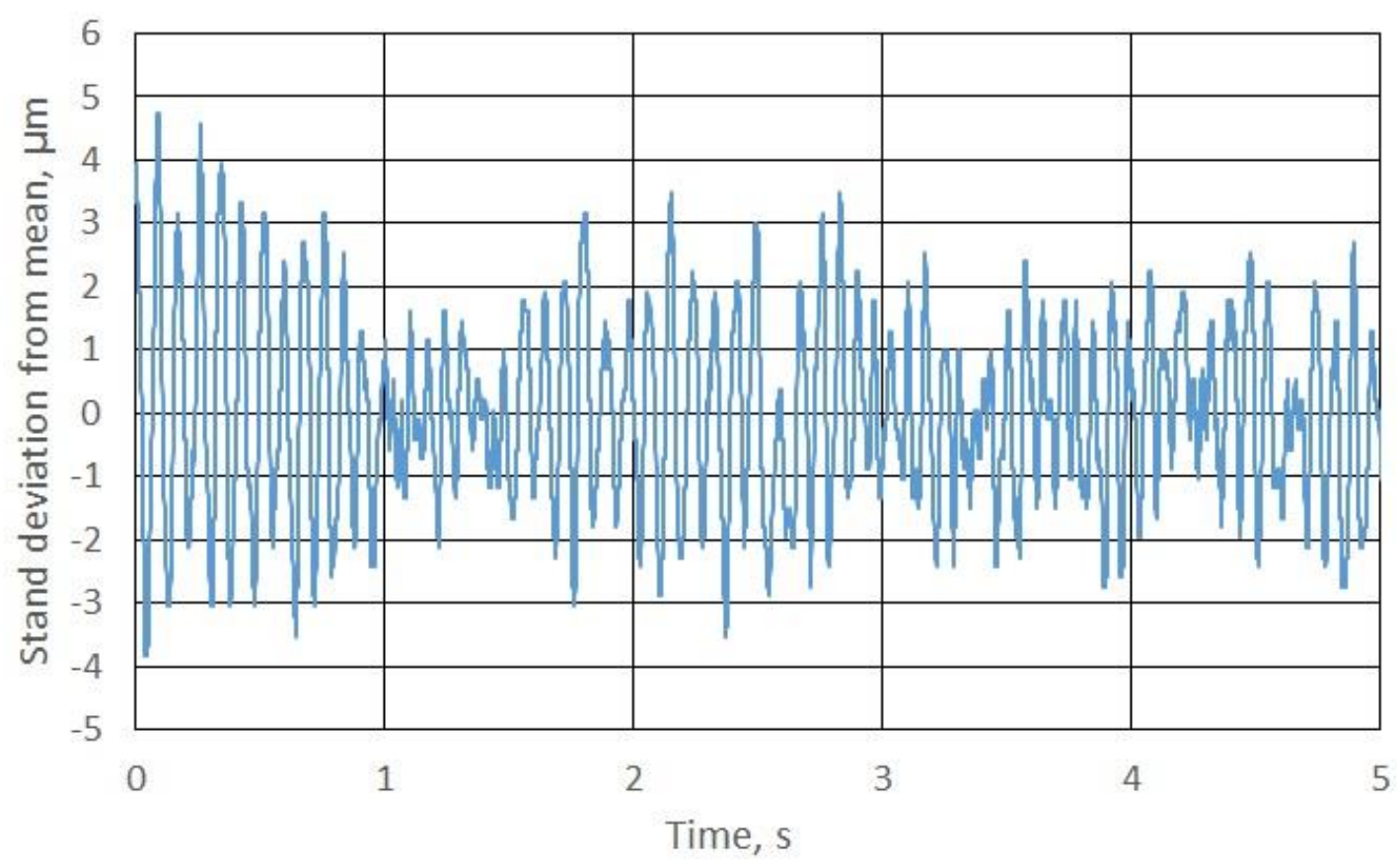

\section{Stand Velocity $\dot{x}=0.3 \mathrm{~mm} / \mathrm{s}$}

Total Mass $m_{\text {stand }}+m_{\text {thruster }}=(14.9+46.7) \mathrm{kg}$ 


\section{Summary of Assumed Values}

\begin{tabular}{l|c|l}
\hline Parameter & Term & Assumed Value \\
\hline TDU Mass & $m_{T D U}$ & $46.7 \mathrm{~kg}$ \\
\hline Stand Mass & $m_{S t a n d}$ & $14.9 \mathrm{~kg}$ \\
\hline Total Weight & $m g$ & $604 \mathrm{~N}$ \\
\hline Stand natural frequency & $\omega_{n}$ & $0.788 \mathrm{~Hz}$ \\
\hline Stand damping coefficient & $c$ & $1.5 \mathrm{~kg} / \mathrm{s}$ \\
\hline Stand position drift & $\left|x_{T}-\widetilde{x}_{i}\right|$ & $2.4 \mu \mathrm{m}$ \\
\hline Stand velocity drift & $\left|\dot{x}_{T}-\tilde{\dot{x}}_{i}\right|$ & $0.3 \mathrm{~mm} / \mathrm{s}$ \\
\hline Stand inclination drift & $\left|\gamma_{T}-\widetilde{\gamma}_{i}\right|$ & $2.0 \mathrm{arc} \mathrm{seconds}$ \\
\hline Shunt thermal sensitivity & $\alpha$ & $100 \mathrm{ppm} /{ }^{\circ} \mathrm{C}$ \\
\hline Shunt thermal drift & $\left|t_{T}-\widetilde{t}_{i}\right|$ & $10^{\circ} \mathrm{C}$ \\
\hline Thrust vector alignment angle & $\theta$ & $2.0^{\circ}$ \\
\hline Calibration slope repeatability & $S_{b}$ & $1.54 \mathrm{mN} / \mathrm{V}$ \\
\hline DAQ uncertainty & $U_{v_{i}}$ & $600 \mu \mathrm{V}$ \\
\hline Calibration pulley moment & $M$ & $2.7 \mathrm{e}-6 \mathrm{Nm}$ \\
\hline Calibration alignment angle & $\varphi$ & $2.0^{\circ}$ \\
\hline Calibration mass uncertainty & $U_{m_{i}}$ & $0.1 \mathrm{~g}$ \\
\hline Gravity uncertainty & $U_{g}$ & $0.01 \mathrm{~m} / \mathrm{s}^{2}$ \\
\hline
\end{tabular}




\section{Summary of Assumed Values}

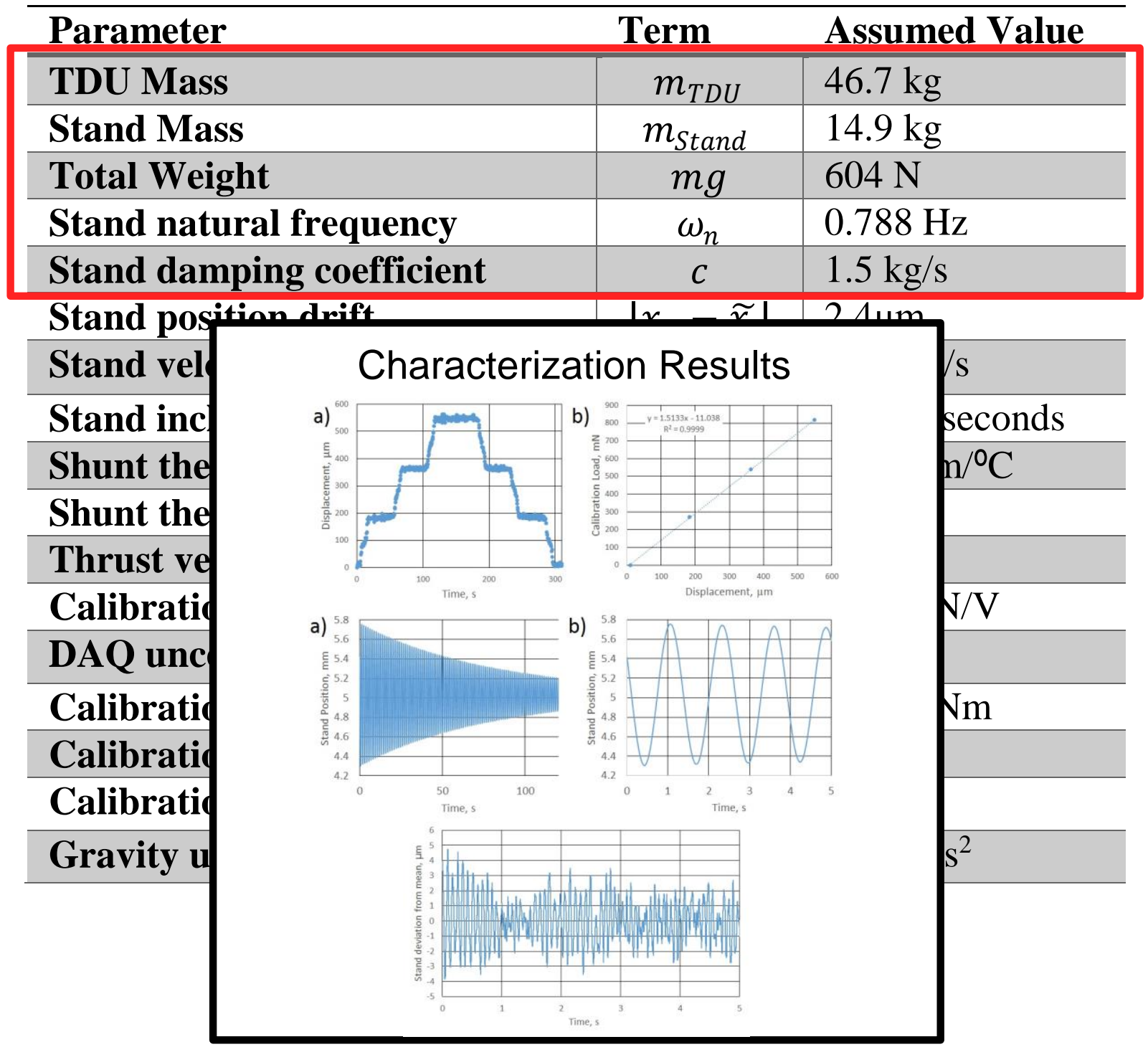




\section{Summary of Assumed Values}

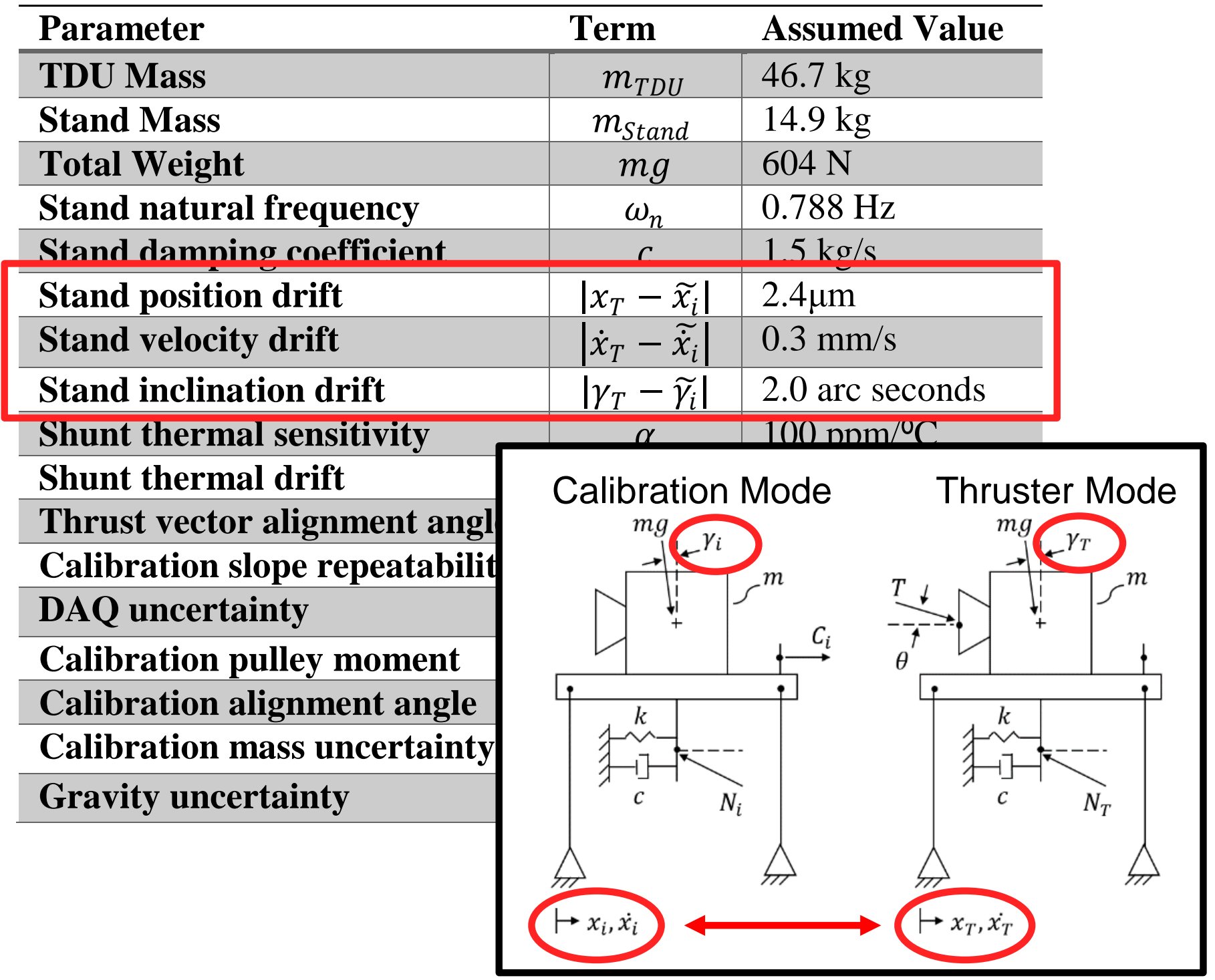




\section{Summary of Assumed Values}

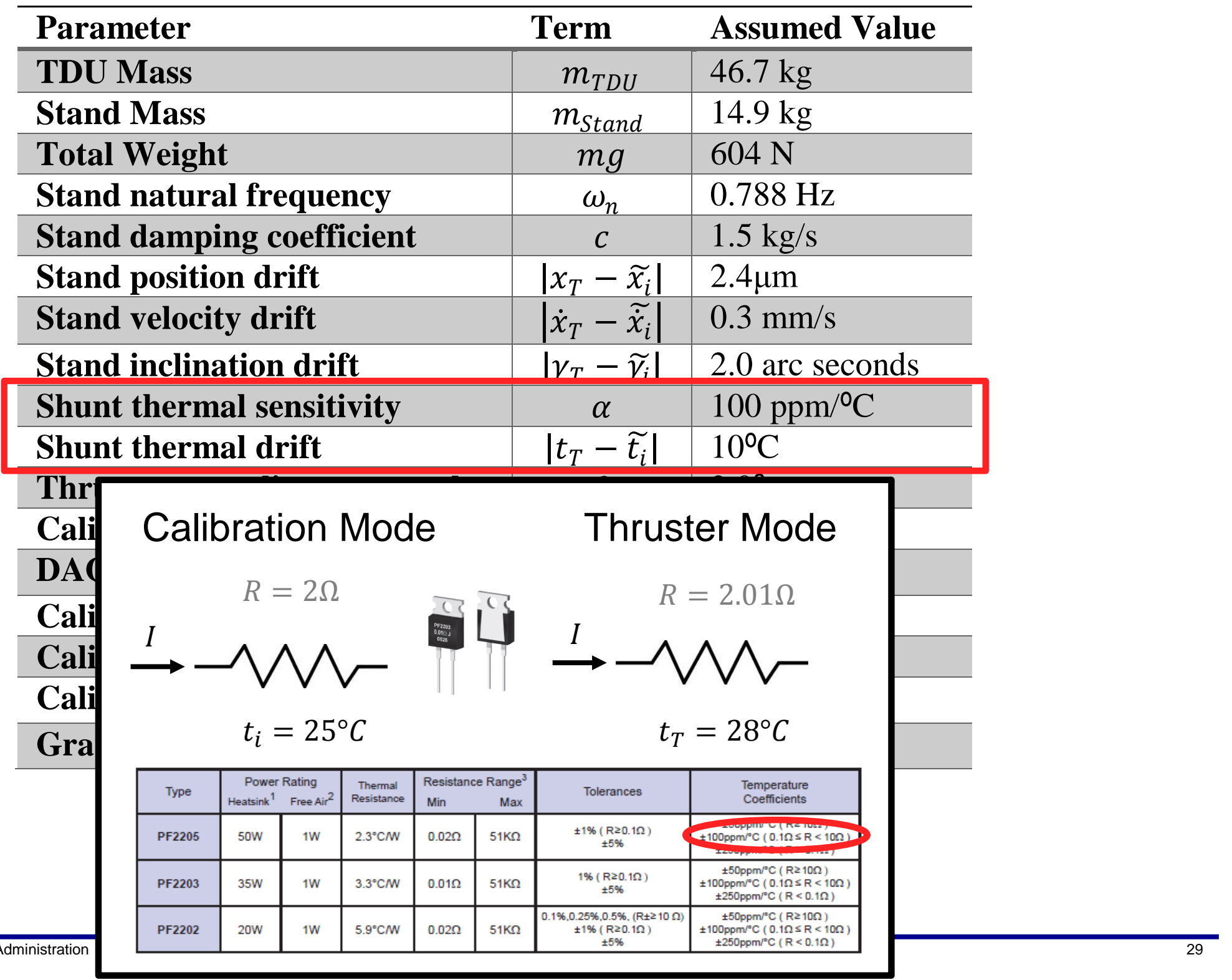




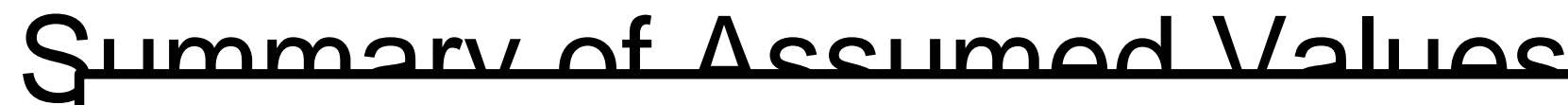

Thruster Mode

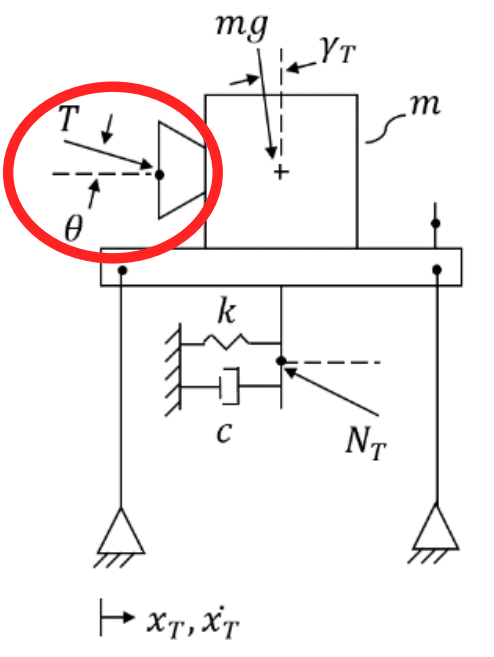

Diagnostic for Verifying the Thrust Vector Requirement of the AEPS Hall-Effect Thruster and Comparison to the

NEXT-C Thrust Vector Diagnostic

Gabriel F. Benavides ${ }^{1}$, Jonathan A. Mackey ${ }^{2}$, Drew M. Ahern ${ }^{3}$, and Robert E. Thomas ${ }^{4}$ NASA Glenn Research Center, Cleveland, OH, USA 44135

\begin{tabular}{|l|c|l|}
\hline Shunt thermal drift & $\left|t_{T}-\widetilde{t}_{i}\right|$ & $10^{\circ} \mathrm{C}$ \\
\hline Thrust vector alignment angle & $\theta$ & $2.0^{\circ}$ \\
\hline Calibration slope repeatability & $S_{b}$ & $1.54 \mathrm{mN} / \mathrm{V}$ \\
\hline DAQ uncertainty & $U_{v_{i}}$ & $600 \mu \mathrm{V}$ \\
\hline Calibration pulley moment & $M$ & $2.7 \mathrm{e}-6 \mathrm{Nm}$ \\
\hline Calibration alignment angle & $\varphi$ & $2.0^{\circ}$ \\
\hline Calibration mass uncertainty & $U_{m_{i}}$ & $0.1 \mathrm{~g}$ \\
\hline Gravity uncertainty & $U_{g}$ & $0.01 \mathrm{~m} / \mathrm{s}^{2}$ \\
\hline
\end{tabular}




\section{Summary of Assumed Values}

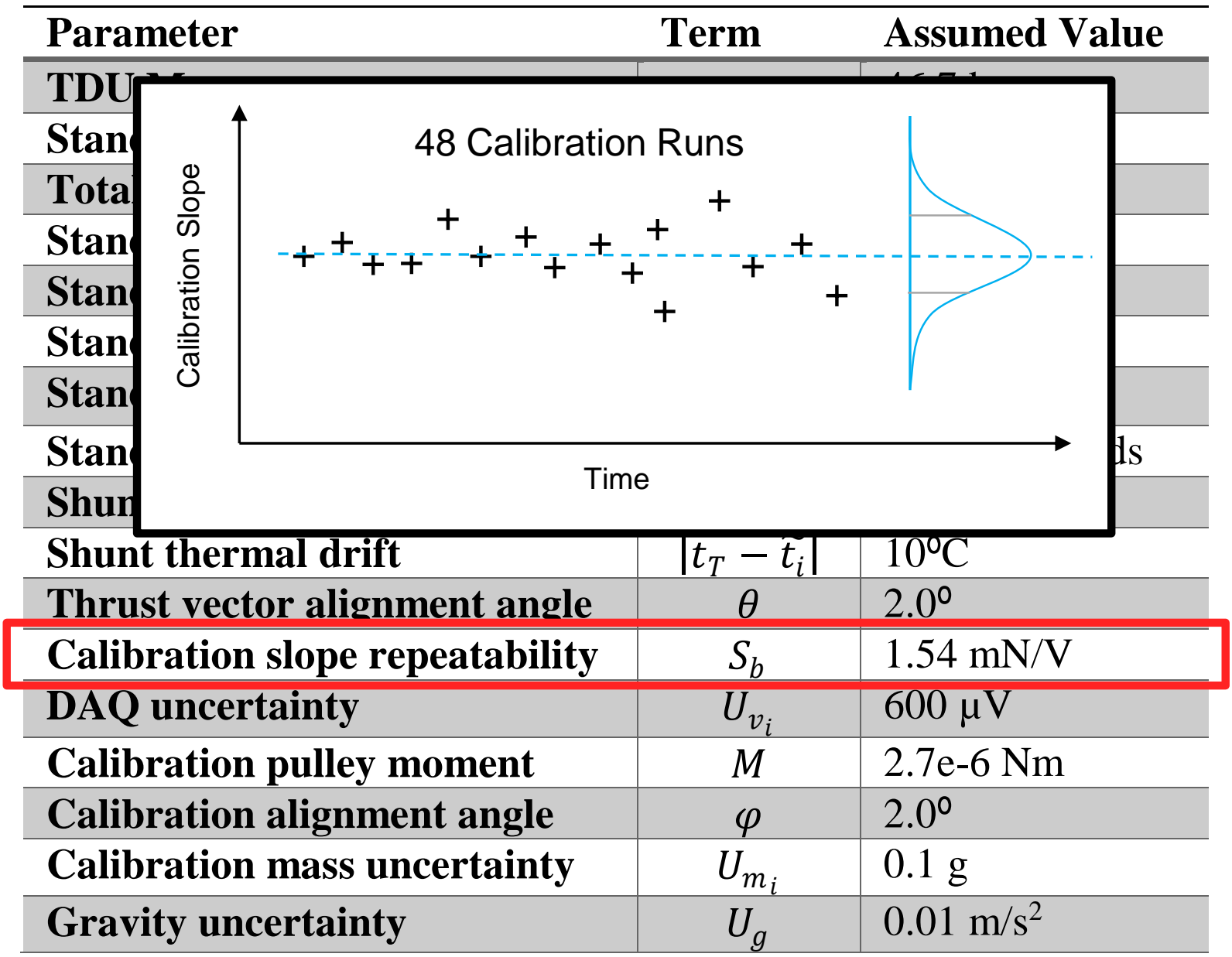




\section{Summary of Assumed Values}

\begin{tabular}{lcc}
\hline Parameter & Term & Assumed Value \\
\hline TDUMass & $m_{m \sim}$ & $46.7 \mathrm{~kg}$ \\
\hline
\end{tabular}

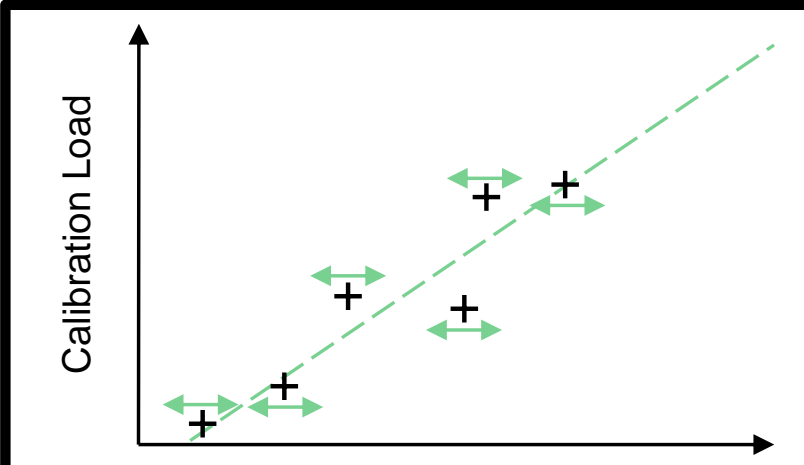

34980 A system specifications and characteristics
DMM accuracy \pm ( $\%$ of reading $+\%$ of range $)$
Includes measurement error, switching error, and transducer conversion error

\begin{tabular}{|c|c|c|c|c|c|c|}
\hline \multirow[b]{2}{*}{ Function } & \multirow[b]{2}{*}{ Range $^{|k|}$} & \multirow[b]{2}{*}{ Frequency, etc. } & \multicolumn{3}{|c|}{ (1) } & \multirow[b]{2}{*}{$\begin{array}{l}\text { Temperature } \\
\text { coefficient } /{ }^{\circ} \mathrm{C} \\
\text { ग Tcal } \pm 5^{\circ} \mathrm{C}\end{array}$} \\
\hline & & & $\begin{array}{l}24 \text { hour }{ }^{[2,3]} \\
\text { Tcal } \pm 1^{\circ} \mathrm{C}\end{array}$ & $\begin{array}{l}90 \text { days } \\
\text { Tcal } \pm 5^{\circ} \mathrm{C}\end{array}$ & $\begin{array}{l}1 \text { year } \\
\text { Tcal } \pm 5^{\circ} \mathrm{C}\end{array}$ & \\
\hline $\begin{array}{l}\text { DC voltage } \\
\text { (with } 34921 \mathrm{~A} / 22 \mathrm{~A} /\end{array}$ & $100.0000 \mathrm{mV}$ & & $\begin{array}{l}0.0030-0.0035 \\
0.0020-0.0006\end{array}$ & $\begin{array}{l}0.0040-0.0040 \\
0.0030-0.0007\end{array}$ & $0.0050+0.0040$ & $0.0005+0.0005$ \\
\hline $31 A / 32 A)$ boulug & & & $0.0015+0.0004$ & $0.0020+0.0005$ & $0.0035+0.0005$ & $0.0005+0.0001$ \\
\hline Input impedance $=\mathrm{Hi}-\mathrm{Z}$ & Tounouour & & $0.003+0.0006$ & $0.0045-0.0006$ & $0.0055=0.0000$ & $0.0000+0.0001$ \\
\hline $10 \mathrm{~V}$ range and below & $300.0000 \mathrm{~V}$ & & $0.003+0.0020$ & $0.0045+0.0030$ & $0.0055-0.0030$ & $0.0005+0.0003$ \\
\hline
\end{tabular}

Shunt Voltage

\begin{tabular}{|l|c|l|}
\hline Shunt thermal drift & $\left|t_{T}-\widetilde{t}_{i}\right|$ & $10^{\circ} \mathrm{C}$ \\
\hline Thrust vector alignment angle & $\theta$ & $2.0^{\circ}$ \\
\hline Calibration slope repeatabilitv & $S_{h}$ & $1.54 \mathrm{mN} / \mathrm{V}$ \\
\hline DAQ uncertainty & $U_{v_{i}}$ & $600 \mu \mathrm{V}$ \\
\hline Calibration pulley moment & $M$ & $2.7 \mathrm{e}-6 \mathrm{Nm}$ \\
\hline Calibration alignment angle & $\varphi$ & $2.0^{\circ}$ \\
\hline Calibration mass uncertainty & $U_{m_{i}}$ & $0.1 \mathrm{~g}$ \\
\hline Gravity uncertainty & $U_{g}$ & $0.01 \mathrm{~m} / \mathrm{s}^{2}$ \\
\hline
\end{tabular}




\section{Summary of Assumed Values}

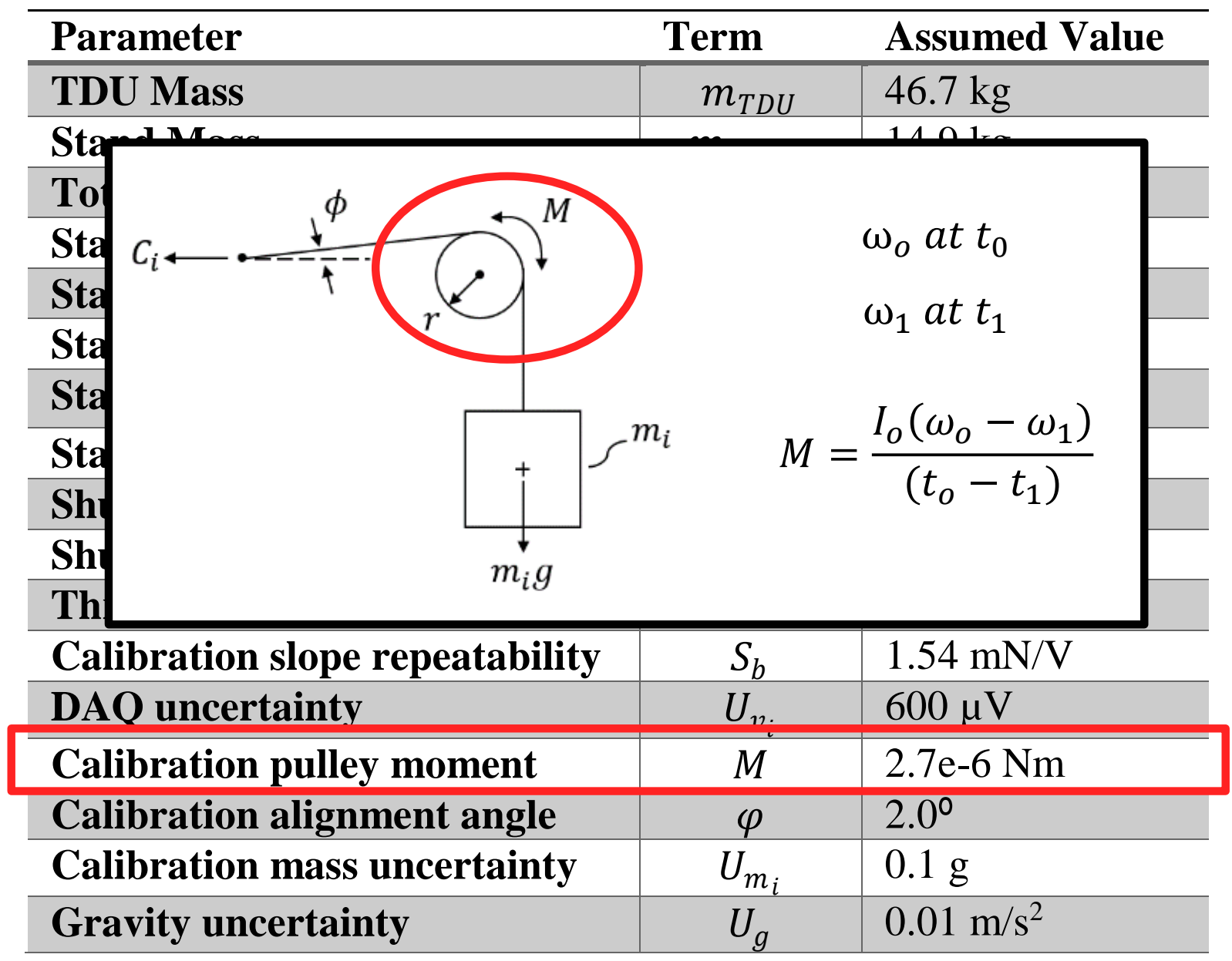




\section{Summary of Assumed Values}

\begin{tabular}{|c|c|c|}
\hline Parameter & Term & Assumed Value \\
\hline TDU Mass & $m_{T D U}$ & $46.7 \mathrm{~kg}$ \\
\hline Stand Mass & $m_{\text {Stand }}$ & $14.9 \mathrm{~kg}$ \\
\hline \multirow{9}{*}{$\begin{array}{l}\text { Conservative } \\
\text { Estimate }\end{array}$} & \multirow{9}{*}{$m_{i} g$} & $04 \mathrm{~N}$ \\
\hline & & $788 \mathrm{~Hz}$ \\
\hline & & $5 \mathrm{~kg} / \mathrm{s}$ \\
\hline & & $4 \mu \mathrm{m}$ \\
\hline & & $3 \mathrm{~mm} / \mathrm{s}$ \\
\hline & & 0 arc seconds \\
\hline & & $\mathrm{p} 0 \mathrm{ppm} /{ }^{\circ} \mathrm{C}$ \\
\hline & & $\mathrm{p}^{\circ} \mathrm{C}$ \\
\hline & & $0^{\underline{0}}$ \\
\hline \multicolumn{3}{|l|}{ Calibration slope tepeataminty } \\
\hline DAQ uncertainty & $U_{v_{i}}$ & $600 \mu \mathrm{V}$ \\
\hline Calibration pullev moment & $M$ & $2.7 \mathrm{e}-6 \mathrm{Nm}$ \\
\hline Calibration alignment angle & $\varphi$ & $2.0^{\circ}$ \\
\hline Calibration mass uncertainty & $U_{m_{i}}$ & $0.1 \mathrm{~g}$ \\
\hline Gravity uncertainty & $U_{g}$ & $0.01 \mathrm{~m} / \mathrm{s}^{2}$ \\
\hline
\end{tabular}




\section{Summary of Assumed Values}

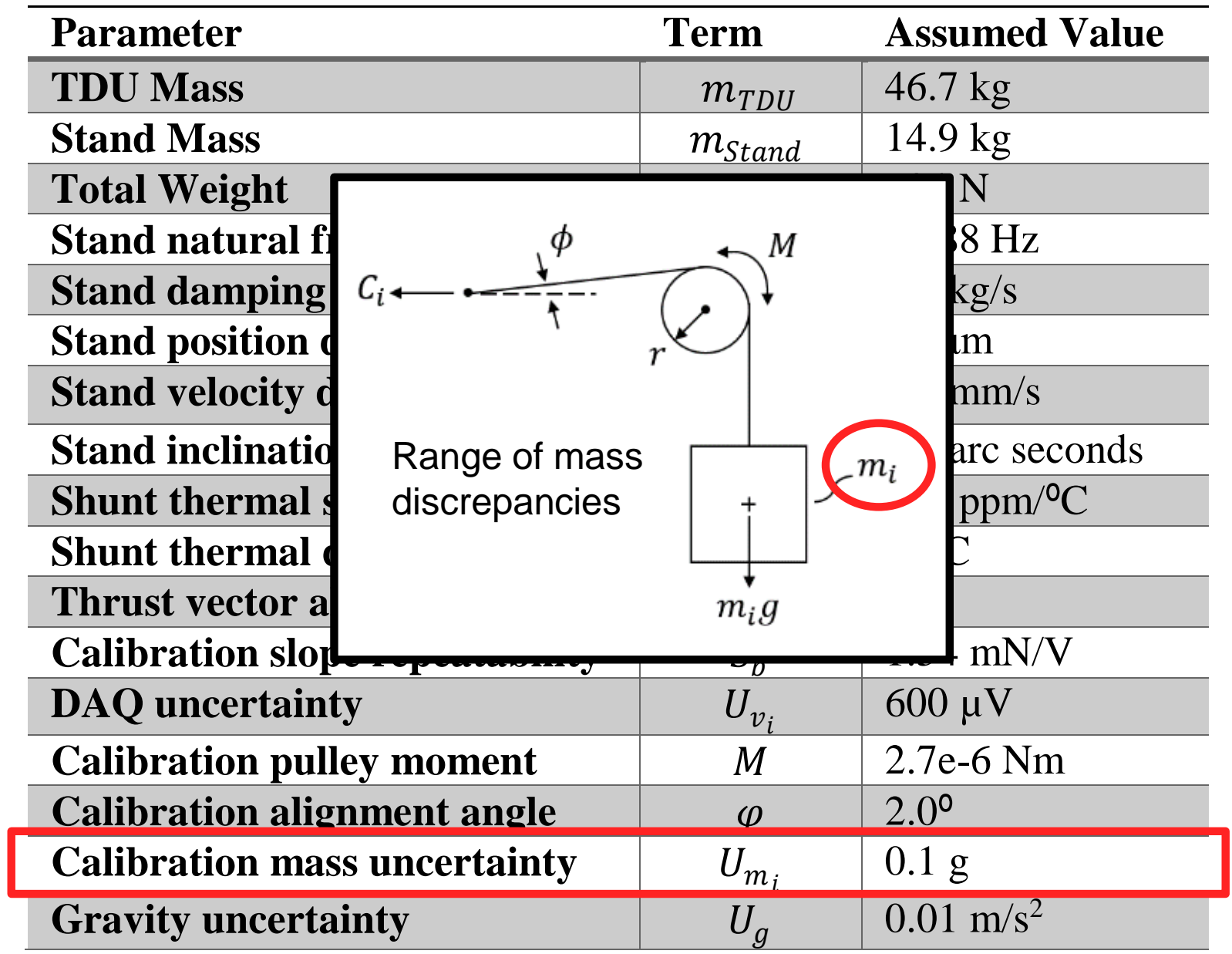




\section{Summary of Assumed Values}

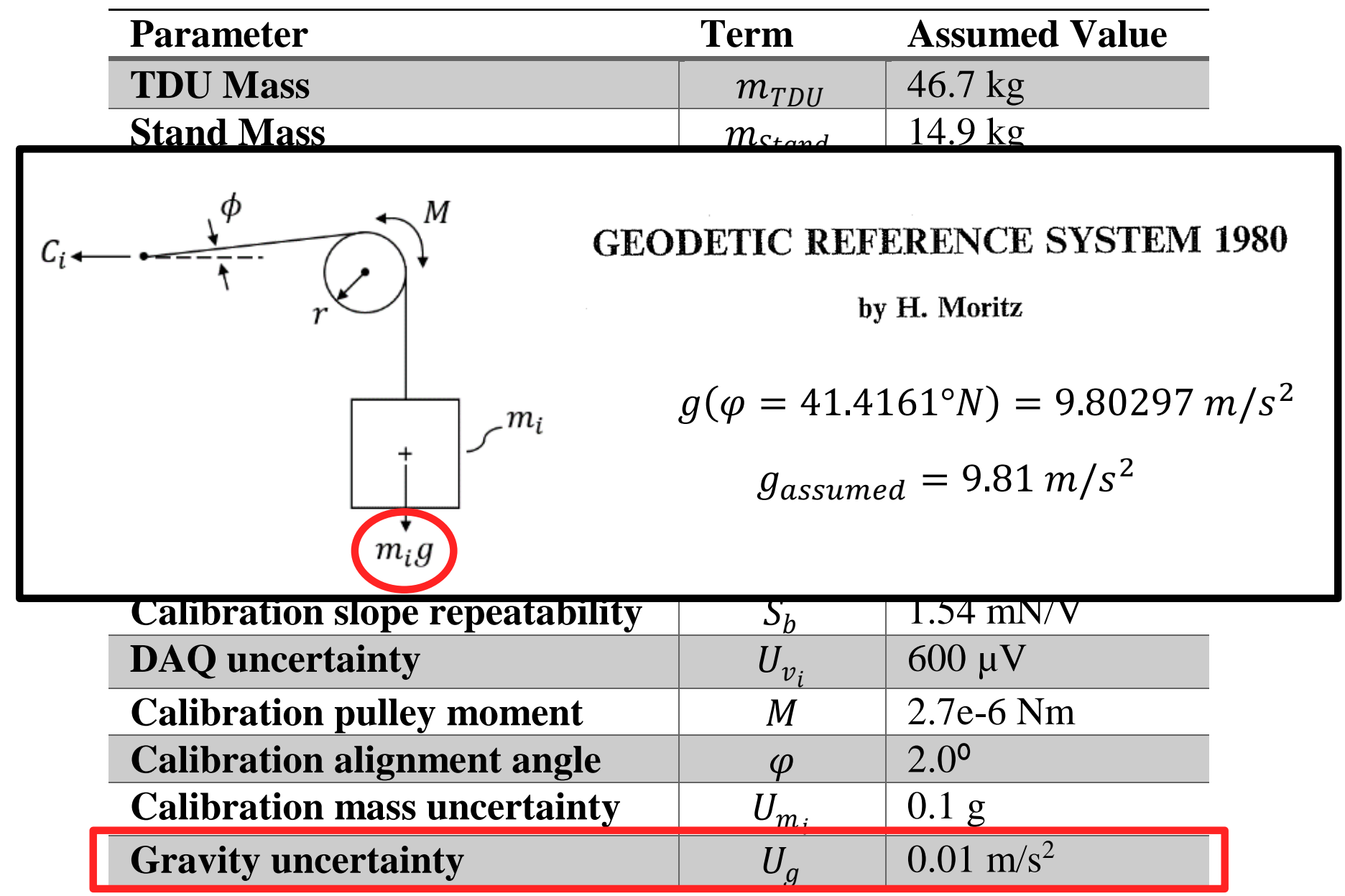




\section{Uncertainty Results}

- Relative uncertainty ranges from 7 to $1 \%$ depending on thrust level.

- TDU generally operates 400 to $600 \mathrm{mN}$, so realistic uncertainty is between 1 to $2 \%$.

- Higher thrust has lower relative uncertainty.

- Absolute uncertainty (also full scale uncertainty) is fairly constant over full range, $\sim 6.9 \mathrm{mN}$.

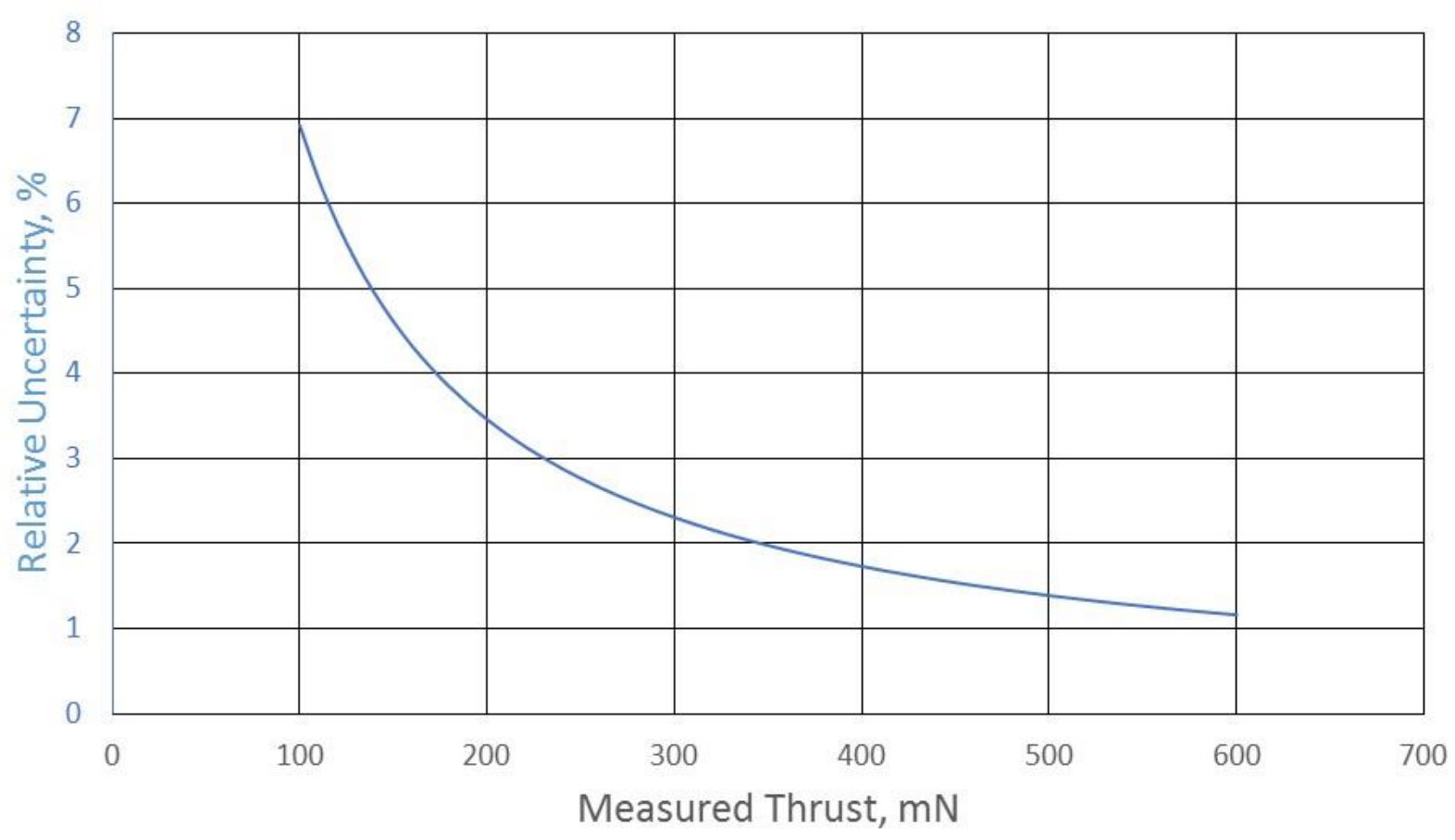




\section{Uncertainty Sources}

- Pareto plots highlight the leading sources of uncertainty for two cases of nominal thrust $(100 \mathrm{mN}$ and $600 \mathrm{mN})$.

- Inclination drift and displacement drift account for $>70 \%$ of the uncertainty.

- Calibration uncertainty, slope repeatability, velocity drift, and DAQ uncertainty are the remaining significant sources.

$100 \mathrm{mN}$

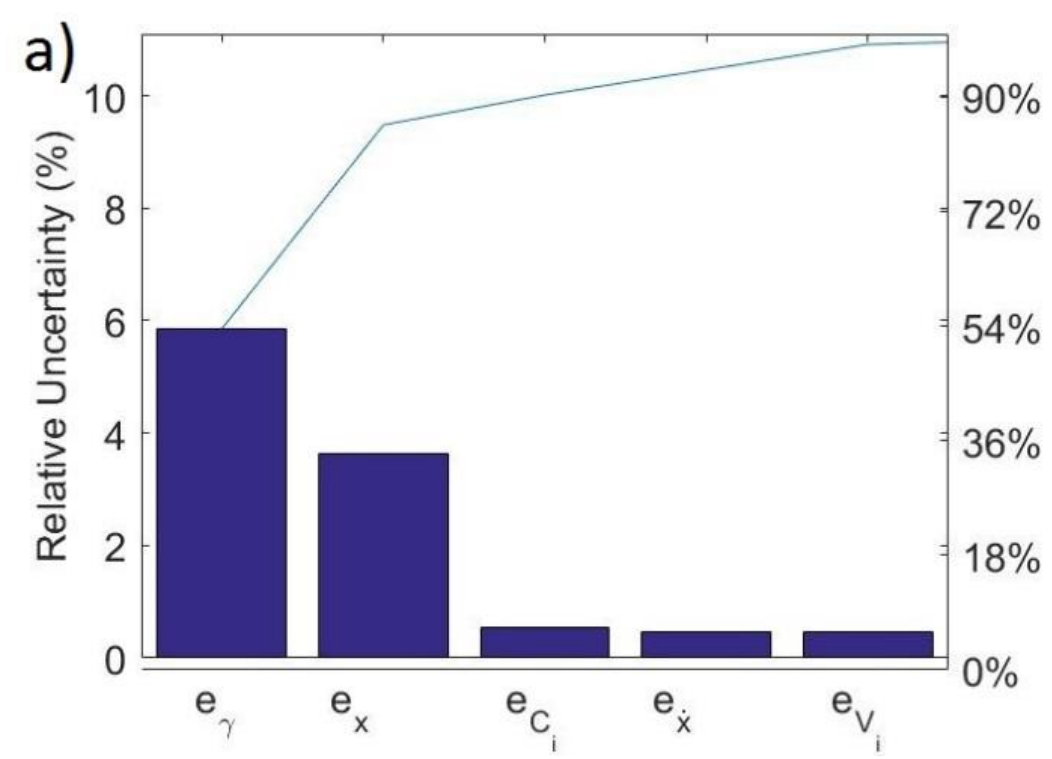

$600 \mathrm{mN}$

b)

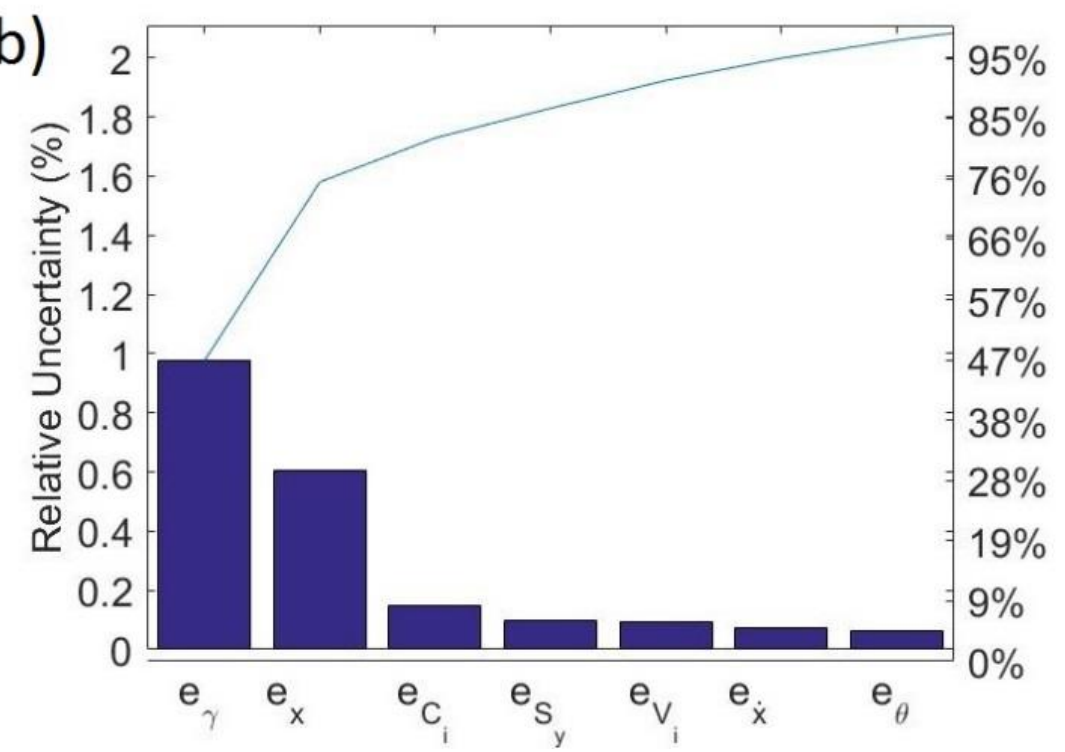




\section{Scaling Parameters}

- Scaling results to other thrust stands, thrusters, and configurations will depend primarily on three scaling factors:

- TDU in VF-6 stiffness to thrust ratio $k / T=2.54 \mathrm{~mm}^{-1}$

- TDU in VF-6 damping to thrust ratio $c / T=2.54 \mathrm{~s} / \mathrm{m}$

- TDU in VF-6 weight to thrust ratio $\mathrm{mg} / \mathrm{T}=1023$

- Considering uncertainty only, it is advisable to minimize these terms.

- Temporal resolution of the stand may suffer for low stiffness ratio.

- Structural integrity of the stand may suffer for low weight ratio. 


\section{Conclusions}

- A $95 \%$ confidence uncertainty of $\pm 6.9 \mathrm{mN}$ has been established for TDU in VF-6.

- The leading sources of uncertainty are stand inclination drift and stand displacement drift.

- A stand characterization method and a set of scaling parameters have been established and calculated for the case study.

- Future Work:

- Various thrusters on VF-6 thrust stand, other inverted pendulum thrust stands, torsional thrust stands, and quantification of additional sources. 


\section{Acknowledgements}

- Space Technology Mission Directorate in support of the Solar Electric Propulsion Technology Demonstration Mission Project for funding the joint NASA GRC and JPL development of the Advanced Electric Propulsion System.

Questions? 
